\title{
MORPHISMS OF SIMPLE TRACIALLY AF ALGEBRAS
}

\author{
MARIUS DADARLAT
}

\begin{abstract}
Let $A, B$ be separable simple unital tracially $\mathrm{AF} \mathrm{C}^{*}$-algebras. Assuming that $A$ is exact and satisfies the Universal Coefficient Theorem (UCT) in KK-theory, we prove the existence, and uniqueness modulo approximately inner automorphisms, of nuclear $*$-homomorphisms from $A$ to $B$ with prescribed K-theory data. This implies the AF-embeddability of separable exact residually finite dimensional $\mathrm{C}^{*}$-algebras satisfying the UCT and reproves Huaxin Lin's theorem on the classification of nuclear tracially AF $\mathrm{C}^{*}$-algebras.
\end{abstract}

\section{INTRODUCTION}

A deep conjecture of Elliott asserts that the simple separable nuclear $\mathrm{C}^{*}$-algebras are classifiable by K-theoretical (and tracial) invariants [18]. While the conjecture is not true is this sweeping level of generality [39], nonetheless it has been proven for large classes of $\mathrm{C}^{*}$-algebras including many remarkable examples. They include the class of simple approximately homogeneous $\mathrm{C}^{*}$-algebras with real rank zero and slow dimension growth [19], [6] $[20,21]$, the Kirchberg $\mathrm{C}^{*}$-algebras [26], [35] and the class of separable simple unital nuclear tracially AF algebras satisfying the UCT [28].

The class of tracially AF algebras was introduced by Lin [29] inspired by [19] and [37]. The tracially AF algebras are characterized by a certain local approximation property, where the approximation is by finite dimensional $\mathrm{C}^{*}$-subalgebras supported on "big" corners. No inductive limit structure is assumed (see Definition 3.1). The added flexibility built into the definition of tracially AF algebras led to important advances [27], [33] and [36] with many more to be expected.

There is an interesting analogy between tracially AF algebras and purely infinite separable simple nuclear algebras (Kirchberg algebras). The algebras in the latter class satisfy a local approximation property similar to the one in the definition of the tracially AF algebras, but with the role of matrix algebras $M_{n}(\mathbb{C})$ played by the Cuntz algebra $\mathcal{O}_{2}$. Elaborating on this analogy, and using relative quasidiagonality as a unifying concept, in our joint work with Eilers [10] we reproved the classification of Kirchberg's algebras satisfying the UCT by the same very method that we used in the proof of the classification of separable simple unital nuclear tracially AF algebras satisfying the UCT with $K_{0}$ group isomorphic to $\mathbb{Q}$.

Kirchberg's Classification Theorem in its most general form [25], [38, Thm. 8.3.3] goes beyond nuclear $\mathrm{C}^{*}$-algebras and proves existence and uniqueness results for nuclear *homomorphisms from $A$ to $B \otimes \mathcal{K}$ with prescribed $\mathrm{KK}_{n u c}$-class for separable unital exact $\mathrm{C}^{*}$ algebras $A$ and unital properly infinite $\mathrm{C}^{*}$-algebras $B$. Recall that $\mathrm{KK}(A, B)=\mathrm{KK}_{n u c}(A, B)$ if $A$ is $\mathrm{K}$-nuclear [42]. It is the goal of this paper to give an analog result for tracially $\mathrm{AF}$ algebras by extending Lin's results of [28]. Let $\underline{\mathrm{K}}(A)$ denote the total K-theory group of

Date: June 29, 2004. 
a $\mathrm{C}^{*}$-algebra $A$, i.e. the direct sum of all $\mathrm{K}$-theory groups of $A$ with integer and torsion coefficients. Our main result is the following.

Theorem 1.1. Let $A, B$ be separable simple unital tracially $A F C^{*}$-algebras. Suppose that $A$ is exact and satisfies the $U C T$. Then for any $\alpha \in \mathrm{KK}(A, B)$ such that the induced map $\alpha_{*}: K_{0}(A) \rightarrow K_{0}(B)$ is order preserving and $\alpha_{*}\left[1_{A}\right]=\left[1_{B}\right]$ there is a nuclear unital *homomorphism $\varphi: A \rightarrow B$ such that $\varphi_{*}(x)=\alpha_{*}(x)$ for all $x \in \underline{\mathrm{K}}(A)$. If $\psi: A \rightarrow B$ is another nuclear $*$-homomorphism with $\psi_{*}=\varphi_{*}: \underline{K}(A) \rightarrow \underline{K}(B)$, then there is a sequence of unitaries $u_{n} \in B$ such that $\lim _{n \rightarrow \infty}\left\|\varphi(a)-u_{n} \psi(a) u_{n}^{*}\right\|=0$ for all $a \in A$.

From Theorem 1.1 and Elliott's intertwining argument one obtains immediately (see Section 6)

Theorem 1.2. (Lin) Let $A, B$ be separable simple unital nuclear tracially $A F C^{*}$-algebras satisfying the UCT. If $\left(K_{0}(A), K_{0}(A)^{+},\left[1_{A}\right]\right) \cong\left(K_{0}(B), K_{0}(B)^{+},\left[1_{B}\right]\right)$ and $K_{1}(A) \cong K_{1}(B)$, then $A$ is isomorphic to $B$.

Another consequence of Theorem 1.1 is the AF-embeddability of separable exact residually finite dimensional $\mathrm{C}^{*}$-algebras satisfying the UCT discussed at the end of Section 6 . Theorem 1.1 was used in a crucial manner in [9] to prove that if a nuclear $\mathrm{C}^{*}$-algebra $A$ admits local approximations by $\mathrm{C}^{*}$-subalgebras satisfying the UCT, then $A$ satisfies the UCT. These subalgebras are necessarily exact but they may be non-nuclear.

The finite $\mathrm{C}^{*}$-algebras are in many respects more rigid than the purely infinite $\mathrm{C}^{*}$-algebras. In particular it is harder to relate $\operatorname{Hom}(A, B)$ to $K K(A, B)$ for tracially AF algebras than for purely infinite algebras. This accounts for why in our main result we were able to interpolate KK-elements by *-homomorphisms only at the level of $\operatorname{Hom}_{\Lambda}(\underline{\mathrm{K}}(A), \underline{\mathrm{K}}(B))$. If $A$ satisfies the UCT, then $\operatorname{Hom}_{\Lambda}(\underline{\mathrm{K}}(A), \underline{\mathrm{K}}(B))$ is the quotient of $K K(A, B)$ by the closure of zero, [5]. Consequently it can serve as a local approximation of $K K(A, B)$. This opens the way for defining partial KK-elements as topological invariants associated with completely positive maps which are approximately multiplicative on large finite sets. Indeed if $\Phi$ is such a map, then it induces a partial map $\Phi_{*}: \underline{\mathrm{K}}(A) \rightarrow \underline{\mathrm{K}}(B)$. The technique of working with approximate morphisms and partial K-theory maps has emerged as one of the key tools in the classification theory of stably finite $\mathrm{C}^{*}$-algebras. (Relative) quasidiagonality also plays a crucial role in the theory as discussed and illustrated in [10]. Indeed, one uses quasidiagonality to go from KK-theory classes to approximate morphisms of $\mathrm{C}^{*}$-algebras.

The classification of tracially AF algebras with arbitrary $K_{0}$ group required an additional technique having to do with finding finite dimensional approximation for the natural map $K_{0}(A) \rightarrow \operatorname{Aff}(T(A))$, and this was discovered by Lin in [28]. In this paper we show that Lin's techniques works equally well for approximating the map $K_{0}(A) \rightarrow \operatorname{Aff} S\left(K_{0}(A)\right)$ for separable unital simple exact $\mathrm{C}^{*}$-algebras with topological tracial rank at most one. We give a new method of handling the infinitesimals of $K_{0}(A)$ and avoid the local approximation by residually finite dimensional $\mathrm{C}^{*}$-algebras. This together with the combined techniques of [10] and [28] leads us to Theorem 1.1.

Having an isomorphism theorem for tracially AF algebras it is natural to exhibit representatives for each isomorphism class. This question was settled by the combined work of Elliott-Gong and Lin. For the sake of completeness we revisit some of the issues relevant to this question. It was shown in [32] that if $A$ is a infinite dimensional separable simple tracially AF $\mathrm{C}^{*}$-algebra then $\left(K_{0}(A), K_{0}(A)^{+},\left[1_{A}\right]\right)$ is a countable weakly unperforated simple scaled ordered group with the Riesz interpolation property and such that $G / G_{\text {tor }}$ is noncyclic. Let 
$\left(G, G^{+}, u\right)$ be a countable weakly unperforated simple scaled ordered group with the Riesz interpolation property and such that $G / G_{t o r}$ is noncyclic. Let $K$ be a countable abelian group. By a result of [19] there is an infinite dimensional separable simple unital tracially $\mathrm{AF}$ algebra $A$, which is actually an inductive limit of homogeneous $\mathrm{C}^{*}$-algebras with spectra of dimension at most three of a special form, such that $\left(K_{0}(A), K_{0}(A)^{+},\left[1_{A}\right]\right) \cong\left(G, G^{+}, u\right)$ and $K_{1}(A) \cong K$. It follows that any separable simple unital nuclear tracially AF $\mathrm{C}^{*}$-algebras satisfying the UCT is isomorphic to some approximately homogeneous $\mathrm{C}^{*}$-algebra. The two Appendices were added for the sake of completness. They contain (largely) self-contained succinct proofs of results due to Elliott-Gong [19] and Lin [28] which are used in the main body of the paper.

\section{Preliminaries}

The UCT and K-theory with coefficients

We begin by recalling the definition of K-theory with mod-m coefficients [41]. Let $\mathbb{T}$ denote the unit circle and let $\mathbb{W}_{m}$ denote the Moore space obtained by attaching the disk to the circle by a degree $m \geq 2$ map. The space $\mathbb{W}_{m}$ constructed in this way is unique up to homotopy equivalence. If $X$ is a compact space we let $C_{0}(X)$ denote the subalgebra of $C(X)$ consisting of functions vanishing at a fixed base point. It is well known that $K_{0}\left(C_{0}\left(W_{m}\right)\right)=\mathbb{Z} / m$ and $K_{1}\left(C_{0}\left(W_{m}\right)\right)=0$. One defines

$$
K_{0}(A ; \mathbb{Z} / m)=K_{0}\left(A \otimes C_{0}\left(\mathbb{W}_{m}\right)\right), \quad K_{1}(A ; \mathbb{Z} / m)=K_{1}\left(A \otimes C_{0}\left(\mathbb{W}_{m}\right)\right) .
$$

It is convenient to work with the following natural realization of K-theory.

$$
\begin{gathered}
K_{*}(A) \cong K_{0}(A \otimes C(\mathbb{T})), \\
K_{*}(A) \oplus K_{*}(A ; \mathbb{Z} / m) \cong K_{0}\left(A \otimes C(\mathbb{T}) \otimes C\left(\mathbb{W}_{m}\right)\right) .
\end{gathered}
$$

The total K-theory group is defined by

$$
\underline{K}(A)=K_{*}(A) \oplus \bigoplus_{m=2}^{\infty} K_{*}(A ; \mathbb{Z} / m) .
$$

The group $\underline{K}(A)$ is acted on by a natural set of coefficient and Bockstein operations, denoted here by $\Lambda[41]$.

Consider the category with objects separable $\mathrm{C}^{*}$-algebras and set of morphisms from $A$ to $B$ given by the Kasparov group $K K(A, B)$. Two $\mathrm{C}^{*}$-algebras that are isomorphic in this category are called KK-equivalent. It was shown by Rosenberg and Schochet [40] that the separable $\mathrm{C}^{*}$-algebras $A$ that are $\mathrm{KK}$-equivalent to abelian $\mathrm{C}^{*}$-algebras are exactly those satisfying the following universal coefficient exact sequence

$$
0 \rightarrow \operatorname{Ext}\left(K_{*}(A), K_{*-1}(B)\right) \rightarrow K K(A, B) \rightarrow \operatorname{Hom}\left(K_{*}(A), K_{*}(B)\right) \rightarrow 0
$$

for any separable $\mathrm{C}^{*}$-algebra $B$. If $A$ has this property we say that $A$ satisfies the UCT.

Theorem 2.1 ([12]). Let $A$ be a separable $C^{*}$-algebra. Then $A$ satisfies the UCT if and only if for any separable $C^{*}$-algebra $B$ there is a short exact sequence

$$
0 \longrightarrow \operatorname{Pext}\left(K_{*}(A), K_{*}(B)\right) \stackrel{\delta}{\longrightarrow} K K(A, B) \stackrel{\Gamma}{\longrightarrow} \operatorname{Hom}_{\Lambda}(\underline{K}(A), \underline{K}(B)) \longrightarrow 0
$$

which is natural in each variable. 
Here Pext denotes the subgroup of Ext $=\operatorname{Ext}_{\mathbb{Z}}^{1}$ consisting of classes of pure extensions. The map $\delta$ is the restriction of the map

$$
\left.\operatorname{Ext}\left(K_{*}(A), K_{*-1}(B)\right)\right) \stackrel{\delta}{\longrightarrow} K K(A, B)
$$

Theorem 2.1 will be referred to as the universal multicoefficient theorem (UMCT) for the Kasparov groups.

\section{Partial maps on K-theory}

Unlike the UCT, the main use of the UMCT is not as much related to the computation of KK groups but rather to the computation of KK-classes of $*$-homomorphisms. Moreover regarding $H_{\mathrm{om}}(\underline{K}(A), \underline{K}(B))$ as an approximation of $K K(A, B)$, the UMCT turns out to be a key technical tool that allows us to work with approximate morphisms of $C^{*}$-algebras. An approximate morphism induces partial maps on K-theory with coefficients as described below. These partial maps will serve as a substitute for the (yet eluding) notion of partial KKtheory element associated to an approximate morphism. But what exactly is an approximate morphism? It is a contractive completely positive (ccp) linear map $\varphi: A \rightarrow B$ which is approximately multiplicative in the following sense. Typically a finite subset $\mathcal{G}$ of $A$ and a number $\delta>0$ are also given. The map $\varphi$ is called $(\mathcal{G}, \delta)$-multiplicative if $\| \varphi\left(a a^{\prime}\right)-$ $\varphi(a) \varphi\left(a^{\prime}\right) \|<\epsilon$ for all $a, a^{\prime} \in \mathcal{G}$. If $\varphi$ is unital and completely positive we say that $\varphi$ is a ucp map. A ccp $(\mathcal{G}, \delta)$-multiplicative map is called a $(\mathcal{G}, \delta)$-approximate morphism or simply an approximate morphism when $(\mathcal{G}, \delta)$ is understood from the context. Let $A, B$, $C$ be $C^{*}$-algebras with $C$ nuclear. If $\varphi: A \rightarrow B$ is a ccp map, we denote by $\widetilde{\varphi}$ the map $\varphi \otimes i d_{C}: A \otimes C \rightarrow B \otimes C$. The following two elementary lemmas are left to the reader.

Lemma 2.2. For any finite set $\widetilde{\mathcal{G}} \subset A \otimes C$ and any $\widetilde{\delta}>0$, there are $\mathcal{G} \subset A$ a finite set and $\delta>0$ such that for any $(\mathcal{G}, \delta)$-multiplicative ccp map $\varphi: A \rightarrow B$, the map $\widetilde{\varphi}$ is $(\widetilde{\mathcal{G}}, \widetilde{\delta})$-multiplicative.

For example let $C=M_{K}(\mathbb{C})$. If $\mathcal{G}$ contains the matrix coefficients of $\widetilde{\mathcal{G}}$ and if $\varphi$ is a $(\mathcal{G}, \delta)$ multiplicative ccp map, then one verifies immediately that $\widetilde{\varphi}$ is $\left(\widetilde{\mathcal{G}}, K^{3} \delta\right)$-multiplicative.

Lemma 2.3. If $a \in A$ satisfies $a \geq 0,\|a\| \leq 1$ and $\left\|a^{2}-a\right\|<1 / 4$, then $\operatorname{Sp}(a) \subset$ $[0,1 / 2) \cup(1 / 2,1]$ and $p=\chi_{(1 / 2,1]}(a)$ is a projection in $A$ with $\|a-p\|<1 / 2$.

We are going to use in an essential manner partial maps on K-theory with coefficients. They are defined as described below. If $A$ is a $\mathrm{C}^{*}$-algebra, we let $\operatorname{Proj}(A)$ denote the set of projections in $\cup_{k=1}^{\infty} M_{k}(A)$ and set

$$
\operatorname{Proj}^{(M)}(A)=\operatorname{Proj}(A \otimes C(\mathbb{T})) \cup \bigcup_{m=2}^{M} \operatorname{Proj}\left(A \otimes C(\mathbb{T}) \otimes C\left(\mathbb{W}_{m}\right)\right) .
$$

We regard $A$ as the subalgebra of constant functions of both $A \otimes C(\mathbb{T})$ and $A \otimes C(\mathbb{T}) \otimes C\left(\mathbb{W}_{m}\right)$ in the obvious way. We write $p \prec q$ if $p, q \in \operatorname{Proj}(A)$ and there is $v \in \cup_{k=1}^{\infty} M_{k}(A)$ with $v^{*} v=p$ and $v v^{*} \leq q$. To an arbitrary ccp map $\varphi: A \rightarrow B$, we associate a map $\varphi_{\sharp}: \operatorname{Proj}(A) \rightarrow K_{0}(B)$ by defining

$$
\varphi_{\sharp}(p)= \begin{cases}{[\chi(\widetilde{\varphi}(p))]} & \text { if }\left\|\widetilde{\varphi}(p)^{2}-\widetilde{\varphi}(p)\right\|<1 / 4, \\ 0 & \text { otherwise, }\end{cases}
$$

where $\chi=\chi_{(1 / 2,1]}$ is the characteristic function of the interval $(1 / 2,1]$ and $\tilde{\varphi}=\varphi \otimes i d$ : $A \otimes \mathcal{K} \rightarrow B \otimes \mathcal{K}$. The map $\varphi_{\sharp}$ is well-defined by Lemma 2.3. In a similar manner we 
associate to $\varphi$ a map $\varphi_{\sharp}: \operatorname{Proj}^{(M)}(A) \rightarrow \underline{\mathrm{K}}(B)$. The only difference is that in the formula (2) we take $\widetilde{\varphi}=\varphi \otimes i d_{C}$ with $C=C(\mathbb{T}) \oplus \bigoplus_{m=2}^{M} C(\mathbb{T}) \otimes C\left(W_{m}\right) \otimes \mathcal{K}$.

Definition 2.4. [10] Let $\mathcal{P} \subset \operatorname{Proj}(A) \cap M_{K}(A)$ and $\mathcal{G} \subset A$ be finite sets and let $\delta>0$. We say that $(\mathcal{P}, \mathcal{G}, \delta)$ is a $K_{0}$-triple if for any ccp map $\varphi: A \rightarrow B$ which is $(\mathcal{G}, \delta)$-multiplicative, the $\operatorname{map} \widetilde{\varphi}=\varphi \otimes \operatorname{id}_{K}: M_{K}(A) \rightarrow M_{K}(B)$ is $(\mathcal{P}, 1 / 4)$-multiplicative, i.e. $\left\|\widetilde{\varphi}(p)^{2}-\widetilde{\varphi}(p)\right\|<1 / 4$ for all $p \in \mathcal{P}$.

More generally if $\mathcal{P} \subset \operatorname{Proj}^{(M)}(A) \cap M_{K}(A \otimes C)\left(\right.$ where $C=C(\mathbb{T}) \oplus\left(\bigoplus_{m=2}^{M} C(\mathbb{T}) \otimes C\left(\mathbb{W}_{m}\right)\right)$ ) and $\mathcal{G} \subset A$ are finite sets and $\delta>0$ we say that $(\mathcal{P}, \mathcal{G}, \delta)$ is a $\underline{K}$-triple if for any ccp map $\varphi: A \rightarrow B$ which is $(\mathcal{G}, \delta)$-multiplicative, the map $\widetilde{\varphi}=\varphi \otimes i d_{M_{K}(C)}$ is $(\mathcal{P}, 1 / 4)$-multiplicative. In this case $\varphi_{\sharp}(p)=[\chi(\widetilde{\varphi}(p))]$ for all $p \in \mathcal{P}$.

Naturally, if $\mathcal{P} \subset \operatorname{Proj}(A)$, then any $\underline{\mathrm{K}}$-triple is implicitly a $K_{0^{0}}$-triple. From Lemma 2.2, we see that for any given finite set $\mathcal{P} \subset \operatorname{Proj}^{(M)}(A)$ there are a finite set $\mathcal{G} \subset A$ and $\delta>0$ such that $(\mathcal{P}, \mathcal{G}, \delta)$ is a K-triple. If $\mathcal{G}^{\prime}$ contains $\mathcal{G}$ and $\delta^{\prime}<\delta$ then $\left(\mathcal{P}, \mathcal{G}^{\prime}, \delta^{\prime}\right)$ is also a K-triple. Moreover if $\mathcal{P}=\left(p_{i}\right)_{i=1}^{\ell}$ and $\mathcal{P}^{\prime}=\left(p_{i}^{\prime}\right)_{i=1}^{\ell}$ are such that $\left[p_{i}\right]=\left[p_{i}^{\prime}\right]$, then we can choose $\mathcal{G}$ and $\delta$ with the property that $\left(\mathcal{P} \cup \mathcal{P}^{\prime}, \mathcal{G}, \delta\right)$ is a $\underline{\mathrm{K}}$-triple and such that for any $(\mathcal{G}, \delta)$-multiplicative $\operatorname{ccp} \operatorname{map} \varphi: A \rightarrow B, \varphi_{\sharp}\left(p_{i}\right)=\varphi_{\sharp}\left(p_{i}^{\prime}\right)$ for $0 \leq i \leq \ell$.

Two sequences $\left(a_{n}\right)$ and $\left(b_{n}\right)$ are called congruent if there is $n_{0}$ such that $a_{n}=b_{n}$ for $n \geq n_{0}$. Congruence is denoted by $\left(a_{n}\right) \equiv\left(b_{n}\right)$ or even $a_{n} \equiv b_{n}$ abusing the notation. If the sequences take values in a group $G$, they are congruent if and only if their image in $\prod_{n} G / \sum_{n} G$ are equal. If $G$ is an ordered group, we say that $a_{n}>0$ asymptotically if $\left(a_{n}\right)$ is congruent to a sequence consisting of strictly positive elements.

We will also work with ccp asymptotic morphisms $\left(\varphi_{n}\right)$. They consists of ccp maps $\varphi_{n}$ : $A \rightarrow B_{n}$ with $\lim _{n \rightarrow \infty}\left\|\varphi_{n}\left(a a^{\prime}\right)-\varphi_{n}(a) \varphi_{n}\left(a^{\prime}\right)\right\|=0$ for all $a, a^{\prime} \in A$. A ccp asymptotic morphism $\left(\varphi_{n}\right)$ induces a sequence of maps $\varphi_{n \sharp}: \operatorname{Proj}^{(M)}(A) \rightarrow \underline{\mathrm{K}}\left(B_{n}\right)$. Note that if $p, q \in$ $\operatorname{Proj}^{(M)}(A)$ have the same class in $\underline{\mathrm{K}}(A)$, then $\varphi_{n \sharp}(p) \equiv \varphi_{n \sharp}(q)$. Assume that $A$ is unital. For any $x \in \underline{\mathrm{K}}(A)$, we fix projections $p, q \in \operatorname{Proj}^{(M)}(A)$ such that $x=[p]-[q]$ and set $\varphi_{n \sharp}(x)=$ $\varphi_{n \sharp}(p)-\varphi_{n \sharp}(q) \in \underline{K}\left(B_{n}\right)$. The sequence $\left(\varphi_{n \sharp}(x)\right)$ depends on the particular projections that we use to represent $x$ but only up to congruence. Indeed if $x=\left[p^{\prime}\right]-\left[q^{\prime}\right]$ for another set of projections $p^{\prime}, q^{\prime}$ then $\left(\varphi_{n \sharp}(p)-\varphi_{n \sharp}(q)\right) \equiv\left(\varphi_{n \sharp}\left(p^{\prime}\right)-\varphi_{n \sharp}\left(q^{\prime}\right)\right)$ since $\left[p \oplus q^{\prime}\right]=\left[p^{\prime} \oplus q\right]$. While in general the maps $\varphi_{n \sharp}: \underline{\mathrm{K}}(A) \rightarrow \underline{\mathrm{K}}\left(B_{n}\right)$ are not group morphisms, the sequence $\left(\varphi_{n \sharp}(x)\right)$ satisfies $\left(\varphi_{n \sharp}(x+y)\right) \equiv\left(\varphi_{n \sharp}(x)+\varphi_{n \sharp}(y)\right)$ for all $x, y \in \underline{K}(A)$. All these properties can be rephrased by saying that $\left(\varphi_{n}\right)$ induces a group morphism

$$
\underline{K}(A) \rightarrow \prod_{n=1}^{\infty} \underline{\mathrm{K}}\left(B_{n}\right) / \sum_{n=1}^{\infty} \underline{\mathrm{K}}\left(B_{n}\right)
$$

Ordered Groups

Let $\left(G, G^{+}, u\right)$ be a simple ordered group with order unit $u$. We write $x \geq 0$ if $x \in G^{+}$ and $x>0$ if $x \in G^{+} \backslash\{0\}$. The state space of $G$ is

$$
S(G)=\left\{f \in \operatorname{Hom}(G, \mathbb{R}): f\left(G^{+}\right) \subset \mathbb{R}^{+}, f(u)=1\right\} .
$$

It is convex and compact in the topology of pointwise convergence. Consider the Banach space $\operatorname{Aff}(S(G))$ of all real valued affine functions on $S(G)$. For $h \in \operatorname{Aff}(S(G)$ ), we write $h>0$ if $h(f)>0$ for all $f \in S(G)$. There is a natural map $\rho: G \rightarrow \operatorname{Aff}(S(G))$ given by $\rho(x)=\widehat{x}$ where $\widehat{x}(f)=f(x)$. The infinitesimals of $G$ is the subgroup of $G$ equal to the 
kernel of $\rho$.

$$
\operatorname{Inf}(G)=\{x \in G: \widehat{x}=0\} .
$$

The group of infinitesimals does not change if we replace $u$ by a different order unit.

Lemma 2.5. Let $\left(G, G^{+}, u\right)$ be a simple ordered group with order unit $u$. Then

$$
\operatorname{Inf}(G)=\{x \in G: \forall n \in \mathbb{Z} \exists m>0, m(u+n x)>0\} .
$$

Proof. This follows from the work of Goodearl and Handelman [22] who showed that if $z \in G^{+}$then

$$
\inf \{f(z): f \in S(G)\}=\sup \{p / q: p u \leq q z, p, q \in \mathbb{N}, q \neq 0\} .
$$

Therefore if $z \in G$, then $m z>0$ for some positive integer $m$ if and only $f(z)>0$ for all $f \in S(G)$. Let $I$ denote the set from the right hand of the equation in the statement. If $x \in \operatorname{Inf}(G)$ and $n \in \mathbb{Z}$, then $f(u+n x)=f(u)=1>0 \forall f \in S(G)$, hence $m(u+n x)>0$ for some $m>0$. This shows that $x \in I$. Conversely if $x \in I, f \in S(G)$ and $n \in \mathbb{Z}$ then $1+n f(x)=f(m(u+n x)) / m \geq 0$ for some $m$. Since this happens for all $n$ we must have $f(x)=0$. This proves that $I \subset \operatorname{Inf}(G)$.

Our main example of a simple ordered group is $\left(G, G^{+}, u\right)=\left(K_{0}(A), K_{0}(A)^{+},\left[1_{A}\right]\right)$ where $A$ is a stably finite simple unital $\mathrm{C}^{*}$-algebra. As another example, recall that if $X$ is a compact connected space and $x_{0} \in X$ is a base point, then $K_{0}(C(X)) \cong \mathbb{Z} \oplus K_{0}\left(C_{0}\left(X \backslash x_{0}\right)\right)$ is a simple ordered group and $\operatorname{Inf} K_{0}(C(X))=K_{0}\left(C_{0}\left(X \backslash x_{0}\right)\right)$ (see [1]).

We collect several results of Blackadar and Handelman [2] and of Effros, Handelman and Shen [16] in the following form (see also Remark 2.7). The terminology not introduced here is taken from [1].

Let $G$ be a countable weakly unperforated ordered group with the Riesz interpolation property. Such a group will be called a generalized dimension group. Let $G$ be a simple generalized dimension group such that $G / G_{\text {tor }}$ is noncyclic. Let $\pi: G \rightarrow G / G_{\text {tor }}$ be the quotient map. If we set $H=G / G_{t o r}$ and $H^{+}=\pi\left(G^{+}\right), H$ is unperforated, it has the Riesz interpolation property and hence it is a dimension group. By results of [16] and [23] we know that $\rho: H \rightarrow \operatorname{Aff}(S(H))$ has dense image and $H$ has the strict ordering induced from the map $\rho$. Since we can identify $S(G)$ with $S(H)$ one obtains the following result.

Theorem 2.6. Let $G$ be a simple generalized dimension group such that $G / G_{t o r}$ is noncyclic. Then

(i) The map $\rho: G \rightarrow \operatorname{Aff}(S(G))$ has uniformly dense range.

(ii) The group $G$ has the strict ordering induced from the map $\rho$. That is

$$
G^{+}=\{0\} \cup\{x \in G: f(x)>0 \quad \forall f \in S(G)\}
$$

(iii) $G / \operatorname{Inf}(G) \cong \rho(G)$ is a noncyclic simple dimension group.

Let us note that as a consequence of (3), the property (ii) above holds for any simple ordered scaled weakly unperforated group $G$. The condition (i) means that $\rho(G)$ is dense in the Banach space $\operatorname{Aff}(S(G))$.

Remark 2.7. Let us exhibit two classes of $\mathrm{C}^{*}$-algebras whose $K_{0}$ groups satisfy the assumptions of the previous theorem. If $A$ is a separable simple stably finite unital $\mathrm{C}^{*}$-algebra, then $\left(K_{0}(A), K_{0}(A)^{+},\left[1_{A}\right]\right)$ is a countable simple ordered group. Generally speaking this group fails to be a generalized dimension group. However this is always the case if in addition we also assume that $A$ has tracial topological rank at most one (in particular if $A$ is tracially 
$\mathrm{AF}$ (see Lemma 3.3 below). On the other hand the Riesz interpolation property also holds for separable stably finite unital $\mathrm{C}^{*}$-algebras $A$ with real rank zero and cancellation of projections [45]. Therefore if $A$ is an infinite dimensional separable simple stably finite unital $\mathrm{C}^{*}$-algebra with with real rank zero, stable rank one and weakly unperforated $K_{0}$ group, then $\left(K_{0}(A), K_{0}(A)^{+},\left[1_{A}\right]\right)$ is a generalized simple dimension group. Moreover $K_{0}(A) / K_{0}(A)_{\text {tor }}$ is noncyclic since $A$ is simple infinite dimensional with real rank zero and hence it has no minimal projections. For future reference we summarize our discussion in the form of a proposition.

Proposition 2.8. Let $A$ be an infinite dimensional separable simple unital $C^{*}$-algebra. Assume that either $A$ has tracial topological rank at most one or that $A$ is stably finite, it has real rank zero and stable rank one and that $K_{0}(A)$ is weakly unperforated. Then

(i) The map $\rho: K_{0}(A) \rightarrow$ Aff $S\left(K_{0}(A)\right)$ has uniformly dense range.

(ii) $K_{0}(A)$ has the strict ordering induced from the map $\rho$. That is

$$
K_{0}(A)^{+}=\{0\} \cup\left\{x \in K_{0}(A): f(x)>0 \quad \forall f \in S\left(K_{0}(A)\right)\right\}
$$

(iii) $K_{0}(A) / \operatorname{Inf} K_{0}(A) \cong \rho\left(K_{0}(A)\right)$ is a noncyclic simple dimension group.

Part (ii) of the proposition holds for separable simple stable finite unital $\mathrm{C}^{*}$-algebras $A$ with with $K_{0}(A)$ weakly unperforated. We are going to use the notation

$$
\operatorname{Inf} \underline{K}(A)=\operatorname{Inf} K_{0}(A) \oplus K_{1}(A) \oplus \bigoplus_{m=2}^{\infty} K_{*}(A ; \mathbb{Z} / m) .
$$

In order to justify this notation, one may note that if $\underline{\mathrm{K}}(A)$ is given the strict ordering induced from the map $\underline{K}(A) \rightarrow K_{0}(A)$, then the infinitesimal subgroup of $\underline{K}(A)$ it is equal to $\operatorname{Inf} \underline{K}(A)$.

\section{LOCAL APPROXIMATIONS}

Definition 3.1. A simple separable unital $\mathrm{C}^{*}$-algebra is called tracially $A F$ if for any finite set $\mathcal{F} \subset A$, any $\epsilon>0$ and any nonzero projection in $p \in A$, there is a nonzero projection $q \in A$ and there is a finite dimensional $\mathrm{C}^{*}$-subalgebra $D \subset q A q$ with $1_{D}=q$ such that

(i) $\|[q, a]\|<\epsilon$ for all $a \in \mathcal{F}$,

(ii) $\operatorname{dist}(q a q, D)<\epsilon$ for all $a \in \mathcal{F}$,

(iii) $q^{\perp}=1-q$ is unitarily equivalent to a subprojection of $p$.

The $\mathrm{C}^{*}$-algebras satisfying the conditions (i) and (ii) were introduced by Popa [37] and are called Popa algebras. It is not hard to check that any hereditary $\mathrm{C}^{*}$-subalgebra of a Popa algebra contains nonzero projections. This shows that the definition of simple tracially AF algebras given above is equivalent to Lin's original definition from [32].

Definition 3.2 ([31]). If in the above definition one relaxes the assumptions on $D$ by requiring $D$ to be an interval $C^{*}$-algebra (rather than finite dimensional), then one says that $A$ has tracial topological rank at most one (written $T R(A) \leq 1$ ). The tracially AF algebras are the $\mathrm{C}^{*}$-algebras of topological rank zero.

An interval $\mathrm{C}^{*}$-algebra is a $\mathrm{C}^{*}$-algebra isomorphic to a finite direct sum of $\mathrm{C}^{*}$-algebras each of which is of the form $M_{k}(\mathbb{C})$ or $M_{k}(C[0,1])$. The $\mathrm{C}^{*}$-algebras with tracial topological rank at most one have a number of useful properties. 
Lemma 3.3 ([31]). Let $A$ be a separable simple unital $C^{*}$-algebra with $T R(A) \leq 1$. Then $A$ is quasidiagonal (hence stably finite) and it has stable rank one. Moreover $K_{0}(A)$ is weakly unperforated and has the Riesz interpolation property. When $p \in A$ is a projection and $n \in \mathbb{N}$, then both $p A p$ and $M_{n}(A)$ are simple unital $C^{*}$-algebras with $T R(A) \leq 1$. If is addition $A$ is tracially $A F$ then it has real rank zero.

In particular, Lemma 3.3 shows that $K_{0}(A)$ is a generalized dimension group.

If $A$ is tracially AF, with the notation from above, define $\omega: A \rightarrow A$ by $\omega(a)=(1-q) a(1-$ $q)$ and $\lambda: A \rightarrow D$ by $\lambda(a)=E(q a q)$ where $E: q A q \rightarrow D$ is a unital conditional expectation. In the case $T R(A) \leq 1$ one defines $E: q A q \rightarrow D$ to be a ucp map with $\|E(x)-x\|<\epsilon_{1}$ for $x$ in a finite subset $\mathcal{F}_{1}$ of $A$ where $\epsilon_{1}$ and $\mathcal{F}_{1}$ will be chosen later.

Lemma 3.4. Let $A$ be a separable simple unital $C^{*}$-algebra with $T R(A) \leq 1$. For any finite set $\mathcal{G} \subset A$, any $\delta>0$ and any nonzero projection in $p \in A$, there is a nonzero projection $q \in A$, there is an interval $C^{*}$-subalgebra $D \subset q A q$ with $1_{D}=q$ and there exists a ucp map $\lambda: A \rightarrow D$ such that if $\omega: A \rightarrow q^{\perp} A q^{\perp}$ is defined by $\omega(a)=q^{\perp} a q^{\perp}$ then

(i) The maps $\omega$ and $\lambda$ are $(\mathcal{G}, \delta)$-multiplicative.

(ii) $\|a-\omega(a)-\lambda(a)\|<\delta, \quad \forall a \in \mathcal{G}$.

(iii) $q^{\perp}$ is unitarily equivalent to a subprojection of $p$.

Proof. Let $\mathcal{F}=\left\{a b: a, b \in \mathcal{G} \cup\left\{1_{A}\right\}\right\}$ and let $\epsilon<\delta /(7 m+5)$ where $m=\sup \{\|a\|: a \in \mathcal{F}\}$. Let $D$ and $q$ be obtained by applying Definition 3.2 to the input data $\mathcal{F}, \delta, p$. In particular for each $a \in \mathcal{F}$ there is $d_{a} \in D$ with $\left\|a-d_{a}\right\|<\epsilon$. Since $D$ is nuclear there is a ucp map $E: q A q \rightarrow D$ such that $\left\|E\left(d_{a}\right)-d_{a}\right\|<\epsilon$ for all $a \in \mathcal{F}$. Indeed, starting with an approximate factorization of $\operatorname{id}_{D}$ by ucp maps $D \stackrel{\alpha}{\mapsto} M_{n}(\mathbb{C}) \stackrel{\beta}{\mapsto} D$, one extends $\alpha$ to a ucp $\operatorname{map} \widetilde{\alpha}: q A q \rightarrow M_{n}(\mathbb{C})$ and defines $E=\beta \widetilde{\alpha}$. If $a \in \mathcal{F}$ then

$$
\|E(q a q)-q a q\| \leq\left\|E(q a q)-E\left(d_{a}\right)\right\|+\left\|E\left(d_{a}\right)-d_{a}\right\|+\left\|d_{a}-a\right\|<3 \epsilon .
$$

Therefore for all $a, b \in \mathcal{G}$

$$
\begin{aligned}
&\|\omega(a b)-\omega(a) \omega(b)\|=\left\|q^{\perp} a\left(b q^{\perp}-q^{\perp} b\right) q^{\perp}\right\|<m \epsilon<\delta . \\
&\|\lambda(a b)-\lambda(a) \lambda(b)\| \leq\|E(q a b q)-E(q a q) E(q b q)\| \\
& \leq\| E(q a b q)-q a b q)\|+\| q a(b q-q b) q \|+ \\
&\|q a q(q b q-E(q b q))\|+\|(q a q-E(q a q)) E(q b q)\| \\
&< 3 \epsilon+\epsilon m+3 \epsilon m+3 \epsilon m=(7 m+3) \epsilon<\delta .
\end{aligned}
$$

Finally we observe that since $\left\|a-q a q-q^{\perp} a q^{\perp}\right\| \leq 2\|q a-a q\|<2 \epsilon$ and $\|E(q a q)-q a q\|<3 \epsilon$, $\|a-\omega(a)-\lambda(a)\|<5 \epsilon<\delta, \quad \forall a \in \mathcal{G}$.

Proposition 3.5. Let $A$ be an separable simple unital $C^{*}$-algebra with $T R(A) \leq 1$. Let $\mathcal{G} \subset A$ be a finite set and let $\delta>0$. There is a sequence $\left(D_{n}\right)$ of mutually orthogonal interval $C^{*}$-subalgebras of $A$ with units $q_{n}=1_{D_{n}}$ satisfying

$$
2^{n}\left[1_{A}-q_{1}-\cdots-q_{n}\right] \leq\left[1_{A}\right] \quad \text { in } K_{0}(A) \text { for all } n \in \mathbb{N}
$$

and there exist two sequences $\left(\lambda_{n}\right)$ and $\left(\omega_{n}\right)$ of ucp maps with $\lambda_{n}: A \rightarrow D_{n}$ and $\omega_{n}: A \rightarrow$ $\left(1_{A}-q_{1}-\cdots-q_{n}\right) A\left(1_{A}-q_{1}-\cdots-q_{n}\right)$ which are $\left(\mathcal{G},\left(1-2^{-n}\right) \delta\right)$-multiplicative and such that

$$
\left\|a-\omega_{n}(a)-\left(\lambda_{1}(a)+\cdots+\lambda_{n}(a)\right)\right\|<\left(1-2^{-n}\right) \delta \quad \text { for all } a \in \mathcal{G} \text { and all } n \in \mathbb{N} \text {. }
$$


Proof. We may assume that $A$ is infinite dimensional. The unit $1_{A}$ of $A$ will be denoted by 1. We proceed by induction. For the first step of induction we let $p_{1}$ be a nonzero projection with $2\left[p_{1}\right] \leq[1]$ in $K_{0}(A)$. Such projections exist by Proposition 2.8. Choose $\delta_{1}>0$ such that $\delta_{1}<2^{-1} \delta$. Let $D_{1}$ and $q_{1}$ be given by Definition 3.2 for the input data $\mathcal{G}, \delta_{1}$ and $p_{1}$. Define ucp maps $\omega_{1}: A \rightarrow\left(1-q_{1}\right) A\left(1-q_{1}\right)$ and $\lambda_{1}: A \rightarrow D_{1}$ as in Lemma 3.4. Then $\omega_{1}$ and $\lambda_{1}$ are $\left(\mathcal{G}, 2^{-1} \delta\right)$-multiplicative. Also $2\left[1-q_{1}\right] \leq 2\left[p_{1}\right] \leq[1]$ and

$$
\left\|a-\omega_{1}(a)-\lambda_{1}(a)\right\| \leq \delta_{1}<2^{-1} \delta \quad \text { for all } a \in \mathcal{G} .
$$

Assume now that $D_{i}, q_{i}, \omega_{i}, \lambda_{i}$ were constructed for $1 \leq i \leq n$ and satisfy the required conditions. We stop here if $q_{1}+\cdots+q_{n}=1$ and take $D_{n+i}=0$ for $i \geq 1$. Otherwise we proceed with the $(n+1)$ th step when we apply the first step for $A_{n}=\left(1-q_{1}-\cdots-q_{n}\right) A\left(1-q_{1}-\cdots-q_{n}\right)$, its finite subset $\omega_{n}(\mathcal{G})$ and the positive number $2^{-n} \delta$. This yields an interval $\mathrm{C}^{*}$-algebra $D_{n+1} \subset A_{n}$ with unit $q_{n+1}$ and ucp maps

$$
\omega^{\prime}: A_{n} \rightarrow\left(1_{A_{n}}-q_{n+1}\right) A\left(1_{A_{n}}-q_{n+1}\right), \quad \lambda^{\prime}: A_{n} \rightarrow D_{n+1}
$$

which are $\left(\omega_{n}(\mathcal{G}), 2^{-n-1} \delta\right)$-multiplicative. Moreover

$2\left[1_{A_{n}}-q_{n+1}\right] \leq\left[1_{A_{n}}\right] \quad$ hence $2^{n+1}\left[1-q_{1}-\cdots-q_{n}-q_{n+1}\right] \leq 2^{n}\left[1-q_{1}-\cdots-q_{n}\right] \leq\left[1_{A}\right]$,

and

$$
\left\|\omega_{n}(a)-\omega^{\prime} \omega_{n}(a)-\lambda^{\prime} \omega_{n}(a)\right\|<2^{-n-1} \delta \quad \text { for all } a \in \mathcal{G} .
$$

Using the identity

$$
\begin{array}{r}
\omega^{\prime}\left(\omega_{n}(a b)\right)-\omega^{\prime}\left(\omega_{n}(a)\right) \omega^{\prime}\left(\omega_{n}(b)\right)=\omega^{\prime}\left(\omega_{n}(a b)-\omega_{n}(a) \omega_{n}(b)\right)+ \\
\omega^{\prime}\left(\omega_{n}(a) \omega_{n}(b)\right)-\omega^{\prime}\left(\omega_{n}(a)\right) \omega^{\prime}\left(\omega_{n}(b)\right)
\end{array}
$$

and $1-2^{n}+2^{-n-1}=1-2^{-n-1}$ we see that the map $\omega_{n+1}=\omega^{\prime} \omega_{n}$ is $\left(\mathcal{G},\left(1-2^{-n-1}\right) \delta\right)$ multiplicative. Similarly $\lambda_{n+1}=\lambda^{\prime} \omega_{n}$ is $\left(\mathcal{G},\left(1-2^{-n-1}\right) \delta\right)$-multiplicative. Moreover

$$
\begin{gathered}
\left\|a-\omega_{n+1}(a)-\left(\lambda_{1}(a)+\cdots+\lambda_{n+1}(a)\right)\right\| \leq\left\|a-\omega_{n}(a)-\left(\lambda_{1}(a)+\cdots+\lambda_{n}(a)\right)\right\| \\
+\left\|\omega_{n}(a)-\omega_{n+1}(a)-\lambda_{n+1}(a)\right\|<\left(1-2^{-n}\right) \delta+2^{-n-1} \delta=\left(1-2^{-n-1}\right) \delta
\end{gathered}
$$

for all $a \in \mathcal{G}$ by (6) and (7). This completes the proof.

\section{Finite dimensional approximations of the Dimension map}

Throughout this section $A$ is a simple unital separable $\mathrm{C}^{*}$-algebra with $T R(A) \leq 1$. The content of this section is based on a idea of Lin [28]. Lin assumes that $A$ is nuclear and bases his arguments on a result of [3] according to which $A$ is an inductive limit of residually finite dimensional $\mathrm{C}^{*}$-algebras. We show that the nuclearity assumption is not needed and make no use of residually finite dimensional $\mathrm{C}^{*}$-algebras. The main result of the section is Proposition 4.4.

The set Aff $S\left(K_{0}(A)\right)$ of all real-valued affine continuous functions on the state space of $K_{0}(A)$ is an ordered Banach space. Recall from Section 2 that there is a natural map dimension map $K_{0}(A) \rightarrow \operatorname{Aff} S\left(K_{0}(A)\right), \rho([p])=\widehat{p}$. The output $\left(D_{n}\right),\left(q_{n}\right),\left(\omega_{n}\right)$ and $\left(\lambda_{n}\right)$ of Proposition 3.5 will be called a $(\mathcal{G}, \delta)$ local approximation of $A$. After breaking $D_{n}$ into elementary direct summands we may assume that $D_{n} \cong M_{k(n)}(\mathbb{C})$ or $D_{n} \cong M_{k(n)}(C[0,1])$. In either case let $D_{n}^{0}=M_{k(n)}(\mathbb{C})$ and let $e_{n}$ be a nonzero minimal projection of $D_{n}^{0}$. The composition of the evaluation map at zero $D_{n} \rightarrow D_{n}^{0}$ with $\lambda_{n}$ is denoted by $\lambda_{n}^{0}: A \rightarrow D_{n}^{0}$. 
If $T R(A)=0$ then of course $D_{n}^{0}=D_{n}$ and $\lambda_{n}^{0}=\lambda_{n}$. The canonical un-normalized trace on $D_{n}$ induces a map $\operatorname{Tr}: K_{0}\left(D_{n}^{0}\right) \rightarrow \mathbb{Z}$ which sends a projection to its trace.

Proposition 4.1. Let $A$ be a separable simple unital $C^{*}$-algebra with $T R(A) \leq 1$. Let $\mathcal{P} \subset M_{K}(A)$ be a finite set of projections and let $\mathcal{G}$ be a finite subset of $A$ that contains the matrix coefficients of all elements of $\mathcal{P}$. If $0<\delta<(4 K)^{-3}$, then for any $(\mathcal{G}, \delta)$ local approximation of $A$ we have

$$
\widehat{p}=\sum_{j=1}^{\infty} \operatorname{Tr} \lambda_{j \sharp}^{0}(p) \widehat{e}_{j}, \quad \text { for all } p \in \mathcal{P} .
$$

The series is convergent in the Banach space Aff $S\left(K_{0}(A)\right)$.

Proof. It is easy to verify that $(\mathcal{P}, \mathcal{G}, \delta)$ is a $K_{0}$-triple using the comment after Lemma 2.2. Thus, if $\widetilde{\lambda}_{n}=\lambda_{n} \otimes i d_{K}$ and $\widetilde{\omega}_{n}=\omega_{n} \otimes i d_{K}$, then $\left\|\widetilde{\lambda}_{n}(p)-\chi\left(\widetilde{\lambda}_{n}(p)\right)\right\|<1 / 2$ and $\| \widetilde{\omega}_{n}(p)-$ $\chi\left(\widetilde{\omega}_{n}(p)\right) \|<1 / 2$ for all $p \in \mathcal{P}$ and $n \in \mathbb{N}$. Since $\mathcal{G}$ contains the matrix coefficients of all elements of $\mathcal{P}$ and $\mathcal{P} \subset M_{K}(A)$, it follows from (6) that

$$
\left\|p-\widetilde{\lambda}_{1}(p)-\cdots-\widetilde{\lambda}_{n}(p)-\widetilde{\omega}_{n}(p)\right\|<K^{2} \delta<1 / 4
$$

hence using Lemma 2.2

$$
\left\|p-\chi\left(\widetilde{\lambda}_{1}(p)\right)-\cdots-\chi\left(\widetilde{\lambda}_{n}(p)\right)-\chi\left(\widetilde{\omega}_{n}(p)\right)\right\|<1 / 2
$$

for all $p \in \mathcal{P}$ and $n \in \mathbb{N}$ since $\left(\lambda_{i}\right)_{i=1}^{n}$ and $\omega_{n}$ have mutually orthogonal ranges. It follows that

$$
[p]=\lambda_{1 \sharp}(p)+\cdots+\lambda_{n \sharp}(p)+\omega_{n \sharp}(p)
$$

in $K_{0}(A)$ for all $p \in \mathcal{P}$ and $n \in \mathbb{N}$. Therefore

$$
\widehat{p}=\widehat{\lambda_{1 \sharp}(p)}+\cdots+\widehat{\lambda_{n \sharp}(p)}+\widehat{\omega_{n \sharp}(p)}
$$

in Aff $S\left(K_{0}(A)\right)$ for all $p \in \mathcal{P}$ and $n \in \mathbb{N}$.

From (5) we have

$$
2^{n}\left[\omega_{n \sharp}(p)\right]=2^{n}\left[\chi\left(\widetilde{\omega}_{n}(p)\right)\right] \leq 2^{n}\left[\widetilde{\omega}_{n}\left(1_{K}\right)\right]=2^{n} K\left[1-q_{1}-\cdots-q_{n}\right] \leq K[1] .
$$

Therefore $\widehat{\omega_{n \sharp}(p)} \leq 2^{-n} K \widehat{1}$, hence $\lim _{n \rightarrow \infty} \widehat{\omega_{n \sharp}(p)}=0$ for all $p \in \mathcal{P}$. We conclude the proof by observing that in formula (9) one has $\lambda_{i \sharp}(p)=\lambda_{i \sharp}^{0}(p)=\operatorname{Tr}\left(\lambda_{i \sharp}^{0}(p)\right)\left[e_{i}\right]$ in $K_{0}(A)$. (Note that $D_{n}^{0}$ is regarded as a $\mathrm{C}^{*}$-subalgebra of $D_{n}$ hence of $A$.)

We need a finite version of formula (8). This is achieved via the following interpolation lemma due to Lin.

Lemma 4.2. Let $X=\left[x_{i j}\right]$ be a matrix in $\operatorname{Mat}_{r \times \infty}(\mathbb{Z})$ with $B=\left[x_{i j}\right]_{1 \leq i, j \leq r}$ nonsingular. Suppose that $\left(e_{j}\right)_{j=1}^{\infty}$ and $\left(v_{i}\right)_{i=1}^{r}$ are sequences in a Banach space $E$ such that

$$
\left.\sum_{j=1}^{\infty} x_{i j} e_{j}=v_{i}, \quad 1 \leq i \leq r, \quad \text { (norm-convergence in } \mathrm{E} .\right)
$$

Then for any $\epsilon>0$ there exist $N \geq r$ and a sequence $\left(h_{j}\right)_{j=1}^{N}$ in $E$ with $\left\|h_{j}-e_{j}\right\|<\epsilon$ for $1 \leq j \leq r, h_{j}=e_{j}$ for $r+1 \leq j \leq N$ and such that

$$
\sum_{j=1}^{N} x_{i j} h_{j}=v_{i}, \quad 1 \leq i \leq r .
$$


Moreover we can arrange that

$$
h_{j} \in \frac{1}{\operatorname{det}(B)}\left(\mathbb{Z} v_{1}+\cdots+\mathbb{Z} v_{r}+\mathbb{Z} e_{r+1}+\cdots+\mathbb{Z} e_{N}\right) \quad \text { for all } 1 \leq j \leq N .
$$

Proof. For any $n>r$ write $X=\left[B\left|C_{n}\right| D_{n}\right]$ where $C_{n} \in \operatorname{Mat}_{r \times(n-r)}(\mathbb{Z})$ and $D_{n} \in \operatorname{Mat}_{r \times \infty}(\mathbb{Z})$. Let $e, v$ be the vector matrices with components $\left(e_{j}\right)_{j=1}^{\infty}$ and $\left(v_{i}\right)_{i=1}^{r}$. Write $e=\left[\begin{array}{c}b \\ c_{n} \\ d_{n}\end{array}\right]$ where $b$ has $r$ components, $c_{n}$ has $n-r$ components and $d_{n}$ is the remaining tail of $e$. Then

$$
X e=B b+C_{n} c_{n}+D_{n} d_{n}=v .
$$

and

$$
\lim _{n \rightarrow \infty}\left\|D_{n} d_{n}\right\|=0 \quad \text { (the norm is taken in } E^{r}=\ell_{r}^{\infty}(E) \text { ) }
$$

since by assumption the series $X e=v$ is norm-convergent. Define

$$
b_{n}=B^{-1}\left(v-C_{n} c_{n}\right) .
$$

If we set $X_{n}=\left[B \mid C_{n}\right]$ and $h=\left[\begin{array}{l}b_{n} \\ c_{n}\end{array}\right]$, then we have

$$
X_{n} h=B b_{n}+C_{n} c_{n}=B B^{-1}\left(v-C_{n} c_{n}\right)+C_{n} c_{n}=v .
$$

Moreover

$$
\left\|b-b_{n}\right\|=\left\|b-B^{-1}\left(v-C_{n} c_{n}\right)\right\|=\left\|b-B^{-1}\left(B b+D_{n} d_{n}\right)\right\| \leq\left\|B^{-1}\right\|\left\|D_{n} d_{n}\right\|,
$$

hence

$$
\lim _{n \rightarrow \infty}\left\|b-b_{n}\right\|=0
$$

by (11). If $\left(h_{j}\right)_{j=1}^{n}$ are the components of $h$, then it follows from (12) and (13) that they satisfy the conclusion of the proposition for some large enough $n=N$.

Proposition 4.3. Let $A$ be a separable simple unital $C^{*}$-algebra with $T R(A) \leq 1$. For any $K_{0}$-triple $(\mathcal{P}, \mathcal{G}, \delta)$, there exist $(\mathcal{G}, \delta)$-multiplicative ucp maps $\lambda_{j}^{0}: A \rightarrow M_{k(j)}(\mathbb{C}), 1 \leq j \leq N$, there are projections $f_{1}, \ldots, f_{N}$ in $\operatorname{Proj}(A)$ and there is a positive integer $R$, such that for all $p \in \mathcal{P}$

$$
\widehat{p}=\frac{1}{R} \sum_{j=1}^{N} \operatorname{Tr} \lambda_{j \sharp}^{0}(p) \widehat{f_{j}} .
$$

Proof. Let $K$ be such that $\mathcal{P} \subset M_{K}(A)$. Without any loss of generality we may assume that $\mathcal{G}$ contains the matrix entries of the elements of $\mathcal{P}$ and $0<\delta<(4 K)^{-3}$. By Proposition 3.5 there is a $(\mathcal{G}, \delta)$ local approximation of $A$. Consequently there exist ucp maps $\left(\lambda_{j}\right)_{j=1}^{\infty}$ and projections $\left(e_{j}\right)_{j=1}^{\infty}$ as in the conclusion of Proposition 4.1.

Thus if $\mathcal{P}=\left\{p_{1}, \ldots, p_{\ell}\right\}$ and $x_{i j}=\operatorname{Tr} \lambda_{j \sharp}^{0}\left(p_{i}\right)$ then

$$
\widehat{p}_{i}=\sum_{j=1}^{\infty} x_{i j} \widehat{e}_{j}, \quad \forall i, 1 \leq i \leq \ell .
$$

If suffices to consider only the case when all $e_{j}$ are nonzero. Indeed, if all but finitely many $e_{j}$ are zero there is nothing to prove. Define $\tilde{\rho}: \mathcal{P} \rightarrow \mathbb{Z}^{\infty} \subset \mathbb{Q}^{\infty}$ by $\tilde{\rho}\left(p_{i}\right)=\left(x_{i 1}, x_{i 2}, x_{i 3}, \ldots\right)$. Let 
$r$ be the dimension of the $\mathbb{Q}$-vector subspace of $\mathbb{Q}^{\infty}$ generated by $\tilde{\rho}(\mathcal{P})$. One has $1 \leq r \leq \ell$. Re-indexing if necessary, we may assume that $\tilde{\rho}\left(p_{1}\right), \ldots, \tilde{\rho}\left(p_{r}\right)$ form a basis of this vector space and that the matrix $B=\left[x_{i j}\right]_{1 \leq i \leq r, 1 \leq j \leq r}$ is nonsingular. Equation (15) shows that if $X=\left[x_{i j}\right]_{1 \leq i \leq r, 1 \leq j<\infty}$ and $e$ is the infinite vector with components $\widehat{e}_{1}, \widehat{e}_{2}, \ldots$, and $v$ has components $\widehat{p}_{1}, \ldots, \widehat{p}_{r}$ then $X e=v$ and the assumptions of Lemma 4.2 are satisfied with $E=\operatorname{Aff} S\left(K_{0}(A)\right)$. Since $A$ is simple, there is $\epsilon>0$ such that $\widehat{e}_{j}>2 \epsilon \widehat{1}$ for all $1 \leq j \leq r$. By Lemma 4.2 we find $N \geq r$ and $h_{1}, \ldots, h_{N} \in \frac{1}{R} \rho\left(K_{0}(A)\right)$ satisfying (10), with $R=|\operatorname{det}(B)|$. Moreover, if we arrange that $\left\|h_{j}-\widehat{e}_{j}\right\|<\epsilon$ for $1 \leq j \leq r$ and $h_{j}=\widehat{e}_{j}$ for $r+1 \leq j \leq N$, then $h_{j} \geq \widehat{e}_{j}-\left\|h_{j}-\widehat{e}_{j}\right\| \widehat{1} \geq \widehat{e}_{j}-\epsilon \widehat{1}>\epsilon \widehat{1}>0$. Therefore $R h_{j} \in \rho\left(K_{0}(A)^{+}\right)$by Proposition 2.8(ii).

This means that there are $f_{j} \in \operatorname{Proj}(A)$ such that $\frac{1}{R} \widehat{f}_{j}=h_{j}$. From (10) we get

$$
\widehat{p}_{i}=\frac{1}{R} \sum_{j=1}^{N} x_{i j} \widehat{f}_{j} \quad \text { for all } 1 \leq i \leq r .
$$

To complete the proof we need to show that in fact (16) holds true for all $i$ with $1 \leq i \leq \ell$ and not just for $1 \leq i \leq r$. Let $s$ be an integer with $1 \leq s \leq \ell$. Since

$$
\tilde{\rho}\left(p_{s}\right) \in \operatorname{span}_{\mathbb{Q}}\left\{\tilde{\rho}\left(p_{1}\right), \ldots, \tilde{\rho}\left(p_{r}\right)\right\} \subset \mathbb{Q}^{\infty},
$$

there are integers $m>0, m_{1}, \ldots, m_{r}$ such that

$$
m \tilde{\rho}\left(p_{s}\right)=\sum_{i=1}^{r} m_{i} \tilde{\rho}\left(p_{i}\right) .
$$

Passing to coordinates we have

$$
m x_{s j}=\sum_{i=1}^{r} m_{i} x_{i j}, \quad 1 \leq j<\infty .
$$

From (15) and (18) we obtain

$$
m \widehat{p}_{s}=\sum_{j=1}^{\infty} m x_{s j} \widehat{e}_{j}=\sum_{j=1}^{\infty}\left(\sum_{i=1}^{r} m_{i} x_{i j}\right) \widehat{e}_{j}=\sum_{i=1}^{r} m_{i}\left(\sum_{j=1}^{\infty} x_{i j} \widehat{e}_{j}\right)=\sum_{i=1}^{r} m_{i} \widehat{p}_{i} .
$$

From (19), (16), and (18) we have for any $1 \leq s \leq \ell$

$$
m \widehat{p}_{s}=\sum_{i=1}^{r} m_{i}\left(\frac{1}{R} \sum_{j=1}^{N} x_{i j} \widehat{f}_{j}\right)=\frac{1}{R} \sum_{j=1}^{N}\left(\sum_{i=1}^{r} m_{i} x_{i j}\right) \widehat{f}_{j}=\frac{1}{R} \sum_{j=1}^{N} m x_{s j} \widehat{f}_{j} .
$$

Dividing by $m$ we obtain the desired conclusion.

If $A$ is a infinite dimensional separable simple unital $\mathrm{C}^{*}$-algebra with $T R(A) \leq 1$, then the image of the map $\rho: K_{0}(A) \rightarrow$ Aff $S\left(K_{0}(A)\right)$ is a dimension group (see Prop. 2.8). Therefore there is an AF algebra $A_{0}$ with $\left(K_{0}\left(A_{0}\right),\left[1_{A_{0}}\right]\right) \cong\left(\rho\left(K_{0}(A)\right), \widehat{1}\right)$.

Proposition 4.4. Let $A$ be a separable simple unital $C^{*}$-algebra with $T R(A) \leq 1$. For any $K_{0}$-triple $(\mathcal{P}, \mathcal{G}, \delta)$ there is a integer $R>0$ and there exists a $(\mathcal{G}, \delta)$-multiplicative ucp map $\psi: A \rightarrow M_{R}\left(A_{0}\right)$ such that after identifying $K_{0}\left(A_{0}\right)$ with $\rho\left(K_{0}(A)\right)$ we have $\psi_{\sharp}(p)=R \widehat{p}$ for all $p \in \mathcal{P}$. 
Proof. We may assume that $1_{A} \in \mathcal{P}$. By Proposition 4.3 there exist $(\mathcal{G}, \delta)$-multiplicative ucp maps $\lambda_{i}: A \rightarrow M_{k(i)}(\mathbb{C}), 1 \leq i \leq N$, projections $f_{1}, \ldots, f_{N}$ in $\operatorname{Proj}(A)$ and a positive integer $R$, such that for all $p \in \mathcal{P}$

$$
R \rho[p]=\sum_{i=1}^{N} \operatorname{Tr} \lambda_{i \sharp}^{0}(p) \rho\left[f_{i}\right] .
$$

Since $1 \in \mathcal{P}$ and $\rho[1]=\left[1_{A_{0}}\right]$, we obtain

$$
\left[1_{M_{R}\left(A_{0}\right)}\right]=R\left[1_{A_{0}}\right]=\sum_{i=1}^{N} \operatorname{Tr} \lambda_{i \sharp}^{0}(1) \rho\left[f_{i}\right]=\sum_{i=1}^{N} k(i) \rho\left[f_{i}\right] .
$$

Let $e_{i}$ be a minimal projection of $M_{k(i)}(\mathbb{C})$. Define an order-preserving homomorphism $\sigma_{*}: K_{0}(D) \rightarrow K_{0}\left(A_{0}\right)=\rho K_{0}(A)$ by $\sigma_{*}\left[e_{i}\right]=\rho\left[f_{i}\right]$. From (21) we see that $\sigma_{*}$ lifts to a unital *-homomorphism $\sigma: D \rightarrow M_{R}\left(A_{0}\right)$. Define

$$
\lambda: A \rightarrow D=M_{k(1)}(\mathbb{C}) \oplus M_{k(2)}(\mathbb{C}) \oplus \cdots \oplus M_{k(N)}(\mathbb{C}),
$$

by $\lambda(a)=\lambda_{1}^{0}(a) \oplus \lambda_{2}^{0}(a) \oplus \cdots \oplus \lambda_{N}^{0}(a)$. Then $\lambda$ is a $(\mathcal{G}, \delta)$-multiplicative ucp map. From

$$
\lambda_{\sharp}\left(p_{j}\right)=\sum_{i=1}^{N} \operatorname{Tr} \lambda_{i \sharp}\left(p_{j}\right)\left[e_{i}\right]
$$

and (20) we obtain

$$
R \rho[p]=(\sigma \lambda)_{\sharp}(p), \quad \text { in } K_{0}\left(A_{0}\right), \quad \forall p \in \mathcal{P} .
$$

We conclude the proof by setting $\psi=\sigma \lambda$.

\section{Approximations on $\operatorname{Inf} \underline{K}(A)$}

The goal of this section is to prove Proposition 5.9. Roughly speaking, we are aiming to lift a given a positive KK element $\alpha$ to an asymptotic morphism which interpolates $\alpha$ on infinitesimals. A key step towards this goal is Thm. 5.5 which exhibits asymptotic morphisms vanishing on infinitesimals. We refer the reader to [24] for a background discussion on Hilbert $\mathrm{C}^{*}$-modules. Let $A, B$ be $\mathrm{C}^{*}$-algebras, let $\mathcal{F} \subset A$ be a finite subset and let $\epsilon>0$. If $\varphi: A \rightarrow \mathcal{L}_{B}(E)$ and $\psi: A \rightarrow \mathcal{L}_{B}(F)$ are two maps, we write $\varphi \underset{\mathcal{F}, \epsilon}{\prec} \psi$ if there is an isometry $v \in \mathcal{L}_{B}(E, F)$ such that $\left\|\varphi(a)-v^{*} \psi(a) v\right\|<\epsilon$ for all $a \in \mathcal{F}$. If $v$ can be chosen to be a unitary, then we write $\varphi \underset{\mathcal{F}_{\epsilon}}{\sim} \psi$. We write $\varphi \prec \psi(\varphi \sim \psi)$ if $\varphi \underset{\mathcal{F}, \epsilon}{\prec} \psi$ (respectively $\varphi \underset{\mathcal{F}_{\epsilon}}{\sim} \psi$ ) for all finite sets $\mathcal{F}$ and for all $\epsilon>0$. Note that if $\varphi \underset{\mathcal{F}_{, \epsilon_{1}}}{\sim} \psi$ and $\psi \underset{\mathcal{F}_{, \epsilon}}{\sim} \gamma$, then $\varphi \underset{\mathcal{F}_{, \epsilon_{1}+\epsilon_{2}}}{\sim} \gamma$. Two ucp asymptotic morphisms $\left(\varphi_{n}\right): A \rightarrow \mathcal{L}_{B}\left(E_{n}\right)$ and $\left(\varphi_{n}^{\prime}\right): A \rightarrow \mathcal{L}_{B}\left(E_{n}^{\prime}\right)$ are called equivalent, $\left(\varphi_{n}\right) \sim\left(\varphi_{n}^{\prime}\right)$ if there is a sequence of unitaries $u_{n} \in \mathcal{L}_{B}\left(E_{n}, E_{n}^{\prime}\right)$ such that $\left\|\varphi_{n}(a)-u_{n}^{*} \varphi_{n}^{\prime}(a) u_{n}\right\| \rightarrow 0$ for all $a \in A$.

Given a map $\varphi: A \rightarrow \mathcal{L}_{B}(E)$ we denote by $n \varphi$ the map $\oplus_{i=1}^{n} \varphi: A \rightarrow \mathcal{L}_{B}\left(E^{n}\right)$. The following lemma is essentially proved in [8]. The revised version presented below was suggested by [34]. 
Lemma 5.1. Let $A$ be a unital $C^{*}$-algebra, let $\mathcal{F}=\mathcal{F}^{*} \subset A$ be a finite subset consisting of unitaries and let $\epsilon>0$. Suppose that $\varphi: A \rightarrow \mathcal{L}_{B}(E)$ and $\psi: A \rightarrow \mathcal{L}_{B}(F)$ are $(\mathcal{F}, \epsilon)$ multiplicative ucp maps with $\varphi \underset{\mathcal{F}_{\epsilon}}{\prec} \psi$. Then there is a $(\mathcal{F}, \epsilon+2 \sqrt{\epsilon})$-multiplicative ucp map $\varphi^{\prime}: A \rightarrow \mathcal{L}_{B}\left(E^{\prime}\right)$ such that then $\varphi \oplus \varphi^{\prime} \underset{\mathcal{F}, \epsilon+2 \sqrt{\epsilon}}{\sim} \psi$.

Proof. By assumption there is $v \in \mathcal{L}_{B}(E, F)$ with

$$
\left\|\varphi(a)-v^{*} \psi(a) v\right\|<\epsilon, \quad \forall a \in \mathcal{F} .
$$

Let $p$ be the projection $v v^{*}$ and let $p^{\perp}=1-p$. Using the identity

$$
\begin{array}{r}
\left(p^{\perp} \psi(a) p\right)^{*} p^{\perp} \psi(a) p=p\left(\psi\left(a^{*}\right) \psi(a)-\psi\left(a^{*} a\right)\right) p+v\left(\varphi\left(a^{*} a\right)-\varphi\left(a^{*}\right) \varphi(a)\right) v^{*} \\
+v\left(\varphi\left(a^{*}\right)-v^{*} \psi\left(a^{*}\right) v\right) \varphi(a) v^{*}+p \psi\left(a^{*}\right) v\left(\varphi(a)-v^{*} \psi(a) v\right) v^{*}
\end{array}
$$

one verifies that

$$
\left\|p^{\perp} \psi(a) p\right\|<2 \sqrt{\epsilon}, \quad \forall a \in \mathcal{F}
$$

hence

$$
\left\|\psi(a)-p \psi(a) p-p^{\perp} \psi(a) p^{\perp}\right\|=\max \left\{\left\|p^{\perp} \psi(a) p\right\|,\left\|p \psi(a) p^{\perp}\right\|\right\}<2 \sqrt{\epsilon}, \quad \forall a \in \mathcal{F} .
$$

If we define $\varphi^{\prime}: A \rightarrow \mathcal{L}_{B}\left(p^{\perp} F\right)$ by $\varphi^{\prime}(a)=p^{\perp} \psi(a) p^{\perp}$, then

$$
\psi \underset{\mathcal{F}, 2 \sqrt{\epsilon}}{\sim} p \psi p+p^{\perp} \psi p^{\perp} \underset{\mathcal{F}, \epsilon}{\sim} \varphi \oplus \varphi^{\prime},
$$

by (24) and (22). Therefore $\psi \underset{\mathcal{F}, \epsilon+2 \sqrt{\epsilon}}{\sim} \varphi \oplus \varphi^{\prime}$. It remains to prove that $\varphi^{\prime}$ is $(\mathcal{F}, \epsilon+2 \sqrt{\epsilon})$ multiplicative. But this follows from the identity

$$
\varphi^{\prime}(a b)-\varphi^{\prime}(a) \varphi^{\prime}(b)=p^{\perp}(\psi(a b)-\psi(a) \psi(b)) p^{\perp}+\left(p^{\perp} \psi(a) p\right) \psi(b) p^{\perp},
$$

using (23) and the assumption that $\psi$ is $(\mathcal{F}, \epsilon)$-multiplicative.

We need the following three lemmas. The first one is related to Voiculescu's theorem and is due to Nate Brown.

Lemma 5.2. [4] Let $A$ be a separable unital $C^{*}$-algebra, let $\mathcal{F} \subset A$ be a finite set and let $\epsilon>0$. Suppose that $\sigma: A \rightarrow \mathcal{L}(H)$ is a unital faithful representation whose image does not contain nonzero compact operators. If $\varphi: A \rightarrow \mathcal{L}(H)$ is a $(\mathcal{F}, \epsilon)$-multiplicative unital map which is a*-monomorphism modulo the compact operators, then $\varphi \underset{\mathcal{F}, 2 \sqrt{\epsilon}}{\sim} \sigma$.

Lemma 5.3. Let $A$ be a separable unital $C^{*}$-algebra. Let $\left(\varphi_{n}\right),\left(\gamma_{n}\right)$ be ucp asymptotic morphisms with $\varphi_{n}: A \rightarrow M_{k(n)}(\mathbb{C})$ and $\gamma_{n}: A \rightarrow M_{r(n)}(\mathbb{C})$. Suppose that $\left(\gamma_{n}\right)$ is faithful in the sense that $\lim \sup _{n}\left\|\gamma_{n}(a)\right\|=\|a\|$ for all $a \in A$. Then there exist a sequence $(\omega(n))$ of disjoint finite subsets of $\mathbb{N}$ with $\max \omega(n-1)<\min \omega(n)$ and a ucp asymptotic morphism $\left(\varphi_{n}^{\prime}\right)$ with $\varphi_{n}^{\prime}: A \rightarrow M_{s(n)}(\mathbb{C})$ such that $\left(\varphi_{n} \oplus \varphi_{n}^{\prime}\right) \sim\left(\gamma_{\omega(n)}\right)$; here $\gamma_{\omega(n)}$ stands for $\bigoplus_{i \in \omega(n)} \gamma_{i}$.

Proof. Since $\left(\varphi_{n}\right)$ is an asymptotic morphism, there exist a sequence $\mathcal{F}_{1} \subseteq \mathcal{F}_{2} \subseteq \cdots$ of finite selfadjoint subsets of $A$ consisting of unitaries whose union is dense in $U(A)$ and a sequence $\epsilon_{1} \geq \epsilon_{2} \geq \cdots$ convergent to zero such that $\varphi_{n}$ is $\left(\mathcal{F}_{n}, \epsilon_{n}\right)$-multiplicative. In order to prove the lemma, it suffices to construct a sequence $(\omega(n))$ such that $\varphi_{n} \underset{\mathcal{F}_{n}, \epsilon_{n}}{\prec} \gamma_{\omega(n)}$. Indeed, Lemma 5.1 will then give $\left(\mathcal{F}_{n}, \delta_{n}\right)$-multiplicative ucp maps $\varphi_{n}^{\prime}$ with $\varphi_{n} \oplus \varphi_{n}^{\prime} \underset{\mathcal{F}_{n}, \delta_{n}}{\sim}$ $\gamma_{\omega(n)}$ where $\delta_{n}=\epsilon_{n}+2 \sqrt{\epsilon_{n}}$. Suppose that $\omega(1), \ldots, \omega(n-1)$ were constructed and choose 
$m>\max \omega(n-1)$ such that $\Gamma=\oplus_{i \geq m} \gamma_{i}: A \rightarrow \prod_{i>m} M_{r(i)}(\mathbb{C}) \subset \mathcal{L}(H)$ is $\left(\mathcal{F}_{n}, \epsilon_{n}^{2} / 4\right)$ multiplicative. Note that when regarded as a map into $\mathcal{L}(H), \Gamma$ is a unital $*$-monomorphism modulo the compact operators. If $\pi: A \rightarrow \mathcal{L}(H)$ is a faithful unital representation with $\pi(A) \cap \mathcal{K}(H)=\{0\}$, then $\Gamma \underset{\mathcal{F}_{n}, \epsilon_{n}}{\sim} \pi$ by Lemma 5.2. If $\pi_{n}$ is the Stinespring dilation of $\varphi_{n}$, then $\pi_{n} \oplus \pi \sim \pi$ by Voiculescu's theorem [43]. Therefore

$$
\varphi_{n} \prec \pi_{n} \prec \pi_{n} \oplus \pi \sim \pi \underset{\mathcal{F}_{n}, \epsilon_{n}}{\sim} \Gamma,
$$

hence $\varphi_{n} \underset{\mathcal{F}_{n}, \epsilon_{n}}{\prec} \Gamma$. Since the image of $\varphi_{n}$ is finite dimensional, by an elementary perturbation argument we see that

$$
\varphi_{n} \underset{\mathcal{F}_{n}, \epsilon_{n}}{\prec} \gamma_{m} \oplus \cdots \oplus \gamma_{m+N}
$$

for some $N$. One concludes the proof by setting $\omega(n)=\{m, \ldots, m+N\}$.

Lemma 5.4. Let $A$ be a separable simple unital $C^{*}$-algebra. Let $\left(\varphi_{n}\right)$ be a ucp asymptotic morphism with $\varphi_{n}: A \rightarrow M_{k(n)}(\mathbb{C}) \cong M_{k(n)}\left(\mathbb{C} 1_{A}\right)$. Then there exists a sequence of integers $(r(n))$ with $r(n) \geq k(n)$ and there is a ucp asymptotic morphism $\left(\varphi_{n}^{\prime}\right)$ with $\varphi_{n}^{\prime}: A \rightarrow$ $M_{r(n)-k(n)}(A)$ such that $\left(\varphi_{n} \oplus \varphi_{n}^{\prime}\right) \sim\left(r(n) \operatorname{id}_{\mathrm{A}}\right)$.

Proof. Let $\left(\mathcal{F}_{n}\right)$ and $\left(\epsilon_{n}\right)$ be as in the proof of Lemma 5.3 with $\varphi_{n}\left(\mathcal{F}_{n}, \epsilon_{n}\right)$-multiplicative. Define $\pi: A \rightarrow M(A \otimes \mathcal{K})$ by $\pi(a)=1 \otimes a$. The representation $\pi$ is unitally nuclearly absorbing by [10, Thm 2.22]. Therefore $\varphi_{n} \oplus \pi \sim \pi$ hence $\varphi_{n} \prec \pi$ for all $n$. An elementary perturbation argument shows that there is an integer $r(n)$ such that $\varphi_{n} \underset{\mathcal{F}_{n}, \epsilon_{n}}{\prec} r(n) \operatorname{id}_{A}$. Applying Lemma 5.1 we find a projection $f_{n} \in M_{r(n)}(A)$ and a $\left(\mathcal{F}_{n}, \delta_{n}\right)$-multiplicative ucp map $\varphi_{n}^{\prime}: A \rightarrow \mathcal{L}_{A}\left(f_{n} A^{r(n)}\right)$ such that $\varphi_{n} \oplus \varphi_{n}^{\prime} \underset{\mathcal{F}_{n}, \delta_{n}}{\sim} r(n) \mathrm{id}_{\mathrm{A}}$, where $\delta_{n}=\epsilon_{n}+2 \sqrt{\epsilon_{n}}$. Since the union of $\mathcal{F}_{n}$ is dense in $U(A)$ and $\delta_{n}$ converges to zero, we see that $\left(\varphi_{n}^{\prime}\right)$ is an asymptotic morphism. By construction we have $f_{n} \oplus 1_{k(n)} \sim 1_{r(n)}$. After replacing $\varphi_{n}^{\prime}$ by $\varphi_{n}^{\prime} \oplus k(n) \operatorname{id}_{A}$ and $r(n)$ by $r(n)+k(n)$ we may assume that $\varphi_{n}^{\prime}(1) \sim 1_{r(n)-k(n)}$ hence $\varphi_{n}^{\prime}: A \rightarrow \mathcal{L}_{A}\left(f_{n} A^{r(n)}\right) \cong M_{r(n)-k(n)}(A)$ is a unital map.

Voiculescu [44] has characterized the (separable) quasidiagonal $\mathrm{C}^{*}$-algebras $A$ as those admitting a faithful embedding $\gamma$ into a corona $\mathrm{C}^{*}$-algebra of the form

$$
\prod_{n=1}^{\infty} M_{k(n)}(\mathbb{C}) / \sum_{n=1}^{\infty} M_{k(n)}(\mathbb{C}),
$$

with $\gamma$ liftable to a ccp map $A \rightarrow \prod M_{k(n)}(\mathbb{C})$. Such a lifting can be viewed as a ccp asymptotic morphism $\left(\gamma_{n}\right)$, with $\gamma_{n}: A \rightarrow M_{k(n)}(\mathbb{C})$, which is faithful in the sense that $\lim \sup _{n}\left\|\gamma_{n}(a)\right\|=\|a\|$ for all $a \in A$. In the case of simple $\mathrm{C}^{*}$-algebras one has some control on the behavior of $\left(\gamma_{n}\right)$ on infinitesimals.

Theorem 5.5. Let $A$ be a separable simple unital quasidiagonal $C^{*}$-algebra. There exists a unital ${ }^{*}$-monomorphism $\gamma: A \rightarrow \prod M_{k(n)}(\mathbb{C}) / \sum M_{k(n)}(\mathbb{C})$ liftable to a ucp map $\left(\gamma_{n}\right): A \rightarrow$ $\prod M_{k(n)}(\mathbb{C})$ such that $\gamma_{*}\left(\operatorname{Inf} K_{0}(A)\right)=0$. Equivalently $\gamma_{n \sharp}(x) \equiv 0$ for all $x \in \operatorname{Inf} K_{0}(A)$.

Proof. Since $A$ is simple, in order to prove the theorem it suffices to show that $A$ has the property that for any two finite subsets $\left\{p_{1}, \ldots, p_{r}\right\},\left\{q_{1}, \ldots, q_{r}\right\}$ of $\operatorname{Proj}(A)$ with $\left[p_{i}\right]-\left[q_{i}\right] \in$ $\operatorname{Inf} K_{0}(A)$, any $\mathcal{G} \subset A$ a finite subset and any $\delta>0$ there is a $(\mathcal{G}, \delta)$-multiplicative ucp map $\Gamma: A \rightarrow M_{k}(\mathbb{C})$ such that $\Gamma_{\sharp}\left(p_{i}\right)-\Gamma_{\sharp}\left(q_{i}\right)=0$ for all $1 \leq i \leq r$. We prove this by induction 
on $r$. We may assume that $p_{1}=q_{1}=0$. In view of Voiculescu's result quoted above there is nothing to prove if $r=1$. Assume now that the property holds for some $r \geq 1$ and let us prove that it also holds for $r+1$. If we let $H=\left\{y_{i}=\left[p_{i}\right]-\left[q_{i}\right]: 1 \leq i \leq r\right\}$ then by assumption there exists a ucp asymptotic morphism $\left(\gamma_{n}\right)$ with $\gamma_{n}: A \rightarrow M_{k(n)}(\mathbb{C})$ such that $\gamma_{n \sharp}(y) \equiv 0$ for all $y \in H$. Let $H^{\prime}=H \cup\{x\}$ where $x=\left[p_{r+1}\right]-\left[q_{r+1}\right]$. We are going to focus on $\left(\gamma_{n \sharp}(x)\right)$. Clearly we only need to discuss the case when $\gamma_{n \sharp}(x) \neq 0$ for all but finitely many $n$. If $\gamma_{n \sharp}(x)>0$ for infinitely many $n$ and $\gamma_{n \sharp}(x)<0$ for infinitely many $n$, then by considering combinations of the form $\gamma_{n}^{\prime}=a(n) \gamma_{n} \oplus b(n) \gamma_{m(n)}$ for suitable integers $a(n), b(n)$ and $m(n) \geq n, \gamma_{n}^{\prime}$ will satisfy $\gamma_{n \sharp}^{\prime}(z)=0$ for all $z \in H^{\prime}$. Thus, after replacing $x$ by $-x$ if necessary, we may assume that $\gamma_{n \sharp}(x)<0$ for all but finitely many $n$. Therefore we may assume that there is $n_{0}$ such that

$$
\gamma_{n \sharp}\left(p_{r+1}\right)-\gamma_{n \sharp}\left(q_{r+1}\right)<0
$$

for all $n \geq n_{0}$. Using Lemma 5.4 we find a ucp asymptotic morphism $\left(\gamma_{n}^{\prime}\right): A \rightarrow$ $M_{r(n)-k(n)}(A)$ such that $\left(\gamma_{n} \oplus \gamma_{n}^{\prime}\right) \sim\left(r(n) \operatorname{id}_{A}\right)$ for some sequence $(r(n))$ of integers. It follows that there are unitaries $u_{n} \in M_{r(n)}(A)$ such that $\left\|u_{n}\left(\gamma_{n} \oplus \gamma_{n}^{\prime}\right)(a) u_{n}^{*}-1_{r(n)} \otimes a\right\| \rightarrow 0$ for all $a \in A$. For $z \in H^{\prime}$ this implies $\gamma_{n \sharp}(z)+\gamma_{n \sharp}^{\prime}(z) \equiv r(n) z$, where we regard $\gamma_{n \sharp}(z)$ and $\gamma_{n \sharp}^{\prime}(z)$ as elements of $K_{0}(A)$ via the inclusions $M_{k(n)}(\mathbb{C})=M_{k(n)}\left(\mathbb{C} 1_{A}\right) \subset M_{k(n)}(A)$ and $M_{r(n)-k(n)}(A) \subset M_{r(n)}(A)$. Therefore

$$
\begin{gathered}
\gamma_{n \sharp}^{\prime}(x) \equiv-\gamma_{n \sharp}(x)+r(n) x, \text { and } \\
\gamma_{n \sharp}^{\prime}(y) \equiv r(n) y,
\end{gathered}
$$

for $y \in H$, since $\gamma_{n \sharp}(y)=0$ by hypothesis. Since $A$ is simple, so is $K_{0}(A)$ by [1], hence $-\gamma_{n \sharp}(x)$ is an order unit of $K_{0}(A)$ whenever $\gamma_{n \sharp}(x)<0$. Since $x \in \operatorname{Inf} K_{0}(A)$, by Lemma 2.5 there is a sequence $(\mathrm{N}(\mathrm{n}))$ of positive integers such that

$$
N(n) \gamma_{n \sharp}^{\prime}(x) \equiv N(n)\left(-\gamma_{n \sharp}(x)+r(n) x\right)>0 \text { for all but finitely many } n \text {. }
$$

After replacing $\gamma_{n}^{\prime}$ by $N(n) \gamma_{n}^{\prime}$ and $r(n)$ by $N(n) r(n)$, we may assume that the ucp map $\gamma_{n}^{\prime}: A \rightarrow M_{t(n)}(A)$ satisfies

$$
\gamma_{n \sharp}^{\prime}(x)>0 \text { (for all but finitely many } n \text { ) in } K_{0}(A)
$$

in addition to (26). Therefore we can find $m$ large enough such that $\gamma_{m}^{\prime}$ is $(\mathcal{G}, \delta)$-multiplicative and

$$
\begin{gathered}
\gamma_{m \sharp}^{\prime}\left(p_{i}\right)-\gamma_{m \sharp}^{\prime}\left(q_{i}\right)=r(m)\left(\left[p_{i}\right]-\left[q_{i}\right]\right) \quad \text { for all } 1 \leq i \leq r, \\
\gamma_{m \sharp}^{\prime}\left(p_{r+1}\right)-\gamma_{m \sharp}^{\prime}\left(q_{r+1}\right)>0 .
\end{gathered}
$$

If $n$ is large enough and we define $\Gamma_{n}=\left(\gamma_{n} \otimes \mathrm{id}_{t(m)}\right) \gamma_{m}^{\prime}$, then $\Gamma_{n}$ is a $(\mathcal{G}, \delta)$-multiplicative ucp map and

$$
\begin{aligned}
\Gamma_{n \sharp}\left(p_{i}\right)-\Gamma_{n \sharp}\left(q_{i}\right)= & r(m)\left(\gamma_{n \sharp}\left(p_{i}\right)-\gamma_{n \sharp}\left(q_{i}\right)\right) \equiv 0 \quad \text { for all } 1 \leq i \leq r, \\
& \Gamma_{n \sharp}\left(p_{r+1}\right)-\Gamma_{n \sharp}\left(q_{r+1}\right)>0 .
\end{aligned}
$$

In the last inequality we use the property that $\left(\gamma_{n}\right)$ is faithful since it is unital and $A$ is simple. Finally, using (25) and (27) we find positive integers $a(n)$ and $b(n)$ such that $\Gamma=a(n) \gamma_{n} \oplus b(n) \Gamma_{n}$ is $(\mathcal{G}, \delta)$-multiplicative and satisfies

$$
\Gamma_{\sharp}\left(p_{i}\right)-\Gamma_{\sharp}\left(q_{i}\right)=0, \quad \text { for all } 1 \leq i \leq r+1 .
$$


A sequence $\left(A_{i}\right)_{i=1}^{\infty}$ of $\mathrm{C}^{*}$-subalgebras of $A$ is called exhausting if for any finite set $\mathcal{F} \subset A$ and any $\epsilon>0$ there is $i$ such that $\operatorname{dist}\left(a, A_{i}\right)<\epsilon$ for all $a \in \mathcal{F}$. It is clear that any increasing sequence of $\mathrm{C}^{*}$-subalgebras of $A$ whose union is dense in $A$ is exhausting.

The following result is $\left[10\right.$, Thm. 5.5]. Recall that $\operatorname{KK}_{n u c}(A, B)=K K(A, B)$ if $A$ is K-nuclear. This is certainly the case if $A$ is nuclear or if $A$ satisfies the UCT [42].

Theorem 5.6. Let $A, B$ be unital $C^{*}$-algebras with $A$ separable, quasidiagonal and let $\alpha \in$ $\operatorname{KK}_{n u c}(A, B)$. For any $\underline{\mathrm{K}}$-triple $(\mathcal{P}, \mathcal{G}, \delta)$ there exist ucp nuclear maps

$$
\varphi: A \rightarrow M_{k}(B) \quad \sigma: A \rightarrow M_{s}\left(\mathbb{C} 1_{B}\right)
$$

which are $(\mathcal{G}, \delta)$-multiplicative and satisfy

$$
\varphi_{\sharp}(p)-\sigma_{\sharp}(p)=\alpha_{*}[p]
$$

for all $p \in \mathcal{P}$. We may arrange that $\varphi(1)$ and $\sigma(1)$ are both projections. Moreover, if $A$ admits an exhausting sequence of residually finite dimensional $C^{*}$-subalgebras and if $\epsilon>0$ is given, we can arrange that there is a unital residually finite dimensional subalgebra $D$ of $A$ such that $\mathcal{G} \subseteq{ }_{\epsilon} D$ and the restriction of $\sigma$ to $D$ is a $*$-homomorphism.

The first part of the theorem shows that there are asymptotic morphisms given by ucp nuclear maps $\varphi_{n}: A \rightarrow M_{k(n)}(B), \sigma_{n}: A \rightarrow M_{s(n)}(\mathbb{C})$ such that $\varphi_{n \sharp}(x)-\sigma_{n \sharp}(x) \equiv \alpha_{*}(x)$ for all $x \in \underline{\mathrm{K}}(A)$.

Corollary 5.7. Let $X$ be a connected compact space with base point $x_{0}$ and let $B$ be a unital $C^{*}$-algebra. Then for any $\alpha \in \mathrm{KK}\left(C_{0}(X), B\right)$ there is a ccp asymptotic morphism $\left(\varphi_{n}\right)$ with $\varphi_{n}: C_{0}(X) \rightarrow M_{k(n)}(B)$ such that $\varphi_{n \sharp}(x) \equiv \alpha_{*}(x)$ for all $x \in \underline{\mathrm{K}}\left(C_{0}(X)\right)$.

Proof. A stronger (continuous) version of this result was already proven in [11] using Etheory. However the corollary can be easily derived from Theorem 5.6. Indeed, regard $\alpha$ as an element of $K K(C(X), B)$ and apply the previous theorem to obtain $\varphi_{n}$ and $\sigma_{n}$ with $\varphi_{n \sharp}(x)-\sigma_{n \sharp}(x) \equiv \alpha_{*}(x)$ for all $x \in \underline{\mathrm{K}}\left(C_{0}(X)\right)$. Since $C(X)$ is residually finite dimensional, we can arrange that $\sigma_{n}$ are $*$-homomorphisms. Since $X$ is connected, the restriction of $\sigma_{n}$ to $C_{0}(X)$ vanishes on K-theory hence one can drop $\sigma_{n}$ altogether.

Definition 5.8. A separable stably finite unital $\mathrm{C}^{*}$-algebra $B$ is called excisive if there is a ccp asymptotic morphism $\left(L_{n}\right): B \rightarrow B$ satisfying the following conditions.

(a) $L_{n \sharp}(x) \equiv x$ for all $x \in \operatorname{Inf} \underline{K}(B)$.

(b) For any $x \in K_{0}(B)$ and any nonzero integer $K$ there is a sequence $\left(x_{n}\right)$ in $K_{0}(B)$ such that $x-L_{n \sharp}(x) \equiv K x_{n}$.

(c) $n L_{n \sharp}(1) \leq[1]$ for all $n \geq 1$.

We give examples of excisive tracially AF algebras in Appendix A. Eventually we'll see that all tracially AF algebras classified by Lin's theorem are excisive.

Proposition 5.9. Let $A$ be a separable simple unital quasidiagonal $C^{*}$-algebra and let $B$ be a separable simple stably finite unital excisive $C^{*}$-algebra. Assume that $B$ has cancellation of projections and that $K_{0}(B)$ is weakly unperforated. Then for any nonzero positive integer $K$ and any $\alpha \in \operatorname{KK}_{n u c}(A, B)$ such that the induced map $\alpha_{*}: K_{0}(A) \rightarrow K_{0}(B)$ is order preserving and $\alpha_{*}\left[1_{A}\right]=\left[1_{B}\right]$, there is a asymptotic morphism $\left(\Phi_{n}\right): A \rightarrow B$ where each $\Phi_{n}$ is a nuclear ccp map and such that

(i) $\alpha_{*}(x)-\Phi_{n \sharp}(x) \equiv 0, \forall x \in \operatorname{Inf} \underline{K}(A)$.

(ii) For any $p \in \operatorname{Proj}(A)$ there is a sequence $\left(q_{n}\right)$ in $\operatorname{Proj}(B)$ with $\alpha_{*}(p)-\Phi_{n \sharp}(p) \equiv K\left[q_{n}\right]$. 
Proof. Let $A, B, \alpha$ and $K$ be as in the statement. In order to prove the proposition it suffices to show that for any two finite sets of projections $\mathcal{P}=\left\{P_{1}, \ldots, P_{r}\right\} \cup\left\{Q_{1}, \ldots, Q_{r}\right\} \subset$ $\operatorname{Proj}^{(M)}(A)$ and $\mathcal{P}_{0}=\left\{p_{1}, \ldots, p_{r}\right\} \subset \operatorname{Proj}(A)$ with

$$
\left[P_{i}\right]-\left[Q_{i}\right] \in \operatorname{Inf} \underline{K}(A), \quad \forall i, 0 \leq i \leq r
$$

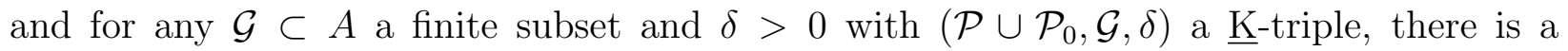
$(\mathcal{G}, \delta)$-multiplicative nuclear ccp map $\Phi: A \rightarrow B$ such that

$$
\begin{gathered}
\alpha_{*}\left(P_{i}\right)-\Phi_{\sharp}\left(P_{i}\right)=\alpha_{*}\left(Q_{i}\right)-\Phi_{\sharp}\left(Q_{i}\right), \quad \forall i, 0 \leq i \leq r \\
\alpha_{*}\left(p_{i}\right)-\Phi_{\sharp}\left(p_{i}\right)=K\left[q_{i}\right], \quad \forall i, 0 \leq i \leq r
\end{gathered}
$$

for some projections $q_{i} \in \operatorname{Proj}(A)$. By Theorem 5.6 there exist asymptotic morphisms $\left(\varphi_{n}\right)$ and $\left(\sigma_{n}\right)$ consisting of nuclear ucp maps $\varphi_{n}: A \rightarrow M_{m(n)}(B)$ and $\sigma_{n}: A \rightarrow M_{s(n)}\left(\mathbb{C} 1_{B}\right)$ such that

$$
\varphi_{n \sharp}(x)-\sigma_{n \sharp}(x) \equiv \alpha_{*}(x) \quad \forall x \in \underline{K}(A) .
$$

Using Theorem 5.5 we find a ucp asymptotic morphism $\left(\gamma_{n}\right)$ with $\gamma_{n}: A \rightarrow M_{k(n)}(\mathbb{C})$ such that

$$
\gamma_{n \sharp}(x) \equiv 0, \quad \text { for all } x \in \operatorname{Inf} \underline{K}(A) .
$$

Indeed, while the vanishing on $\operatorname{Inf} K_{0}(A)$ is given by Theorem $5.5, \gamma_{n \sharp}$ vanishes asymptotically on $K_{1}(A)$ since the range of $\gamma_{n}$ is contained in a finite dimensional $\mathrm{C}^{*}$-algebra. Moreover, by replacing $\gamma_{n}$ by $n ! \gamma_{n}$ we obtain that $\gamma_{n \sharp}$ vanishes asymptotically on K-theory with torsion coefficients .

Since $A$ is simple and $\gamma_{n}$ is unital we have $\lim _{n \rightarrow \infty}\left\|\gamma_{n}(a)\right\|=\|a\|$ for all $a \in A$. We apply Lemma 5.3, to obtain a ucp asymptotic morphism $\left(\sigma_{n}^{\prime}\right)$ and a sequence of sets $(\omega(n))$ such that $\left(\sigma_{n} \oplus \sigma_{n}^{\prime}\right) \sim\left(\gamma_{\omega(n)}\right)$. Therefore $\left(\sigma_{n} \oplus \sigma_{n}^{\prime}\right)_{\sharp}(x) \equiv\left(\gamma_{\omega(n)}\right)_{\sharp}(x)$ hence

$$
\left(\varphi_{n} \oplus \sigma_{n}^{\prime} \oplus(K-1) \gamma_{\omega(n)}\right)_{\sharp}(x)-K\left(\gamma_{\omega(n)}\right)_{\sharp}(z) \equiv \alpha_{*}(x), \quad \forall x \in \underline{K}(A) .
$$

Note that we still have

$$
\left(\gamma_{\omega(n)}\right)_{\sharp}(x)=0, \quad \forall x \in \operatorname{Inf} \underline{K}(A) .
$$

If we define

$$
\varphi=\varphi_{n} \oplus \sigma_{n}^{\prime} \oplus(K-1) \gamma_{\omega(n)}, \quad \gamma=\gamma_{\omega(n)},
$$

and $n$ large enough, then $\varphi: A \rightarrow M_{m}(B)$ and $\gamma: A \rightarrow M_{s}\left(\mathbb{C} 1_{B}\right)$ are $(\mathcal{G}, \delta)$-multiplicative ucp maps such that

$$
\begin{gathered}
\gamma_{\sharp}\left(P_{i}\right)-\gamma_{\sharp}\left(Q_{i}\right)=0, \quad \forall i, 1 \leq i \leq r . \\
\varphi_{\sharp}(P)-K \gamma_{\sharp}(P)=\alpha_{*}[P], \quad \forall P \in \mathcal{P} \cup \mathcal{P}_{0} .
\end{gathered}
$$

Therefore

(33) $\alpha_{*}\left(\left[P_{i}\right]-\left[Q_{i}\right]\right)=\varphi_{\sharp}\left(P_{i}\right)-\varphi_{\sharp}\left(Q_{i}\right)-K\left(\gamma_{\sharp}\left(P_{i}\right)-\gamma_{\sharp}\left(Q_{i}\right)\right)=\varphi_{\sharp}\left(P_{i}\right)-\varphi_{\sharp}\left(Q_{i}\right) \quad \forall i, 1 \leq i \leq r$.

Since $\alpha_{*}$ is a morphism of ordered scaled groups it must preserve the infinitesimals. Hence we obtain from (28) that

$$
\alpha_{*}\left(\left[P_{i}\right]-\left[Q_{i}\right]\right) \in \operatorname{Inf} \underline{K}(B) .
$$

Let $\left(L_{n}\right): M_{m}(B) \rightarrow M_{m}(B)$ be an asymptotic morphism given by Definition 5.8. Then $L_{n \sharp}(x) \equiv x$ for all $x \in \operatorname{Inf} \underline{K}(B)$. Therefore using (33)

$$
\alpha_{*}\left(\left[P_{i}\right]-\left[Q_{i}\right]\right) \equiv L_{n \sharp}\left(\alpha_{*}\left(\left[P_{i}\right]-\left[Q_{i}\right]\right)\right) \equiv\left(L_{n} \varphi\right)_{\sharp}\left(P_{i}\right)-\left(L_{n} \varphi\right)_{\sharp}\left(Q_{i}\right) \quad \forall i, 1 \leq i \leq r .
$$


Thus if we define $\Phi=L_{n} \varphi$ and $n$ is large enough, then $\Phi$ satisfies (29).

Using part (b) of Definition 5.8, for each $p_{i} \in \mathcal{P}_{0}$ we find sequences $\left(x_{i n}\right)_{n}$ in $K_{0}(B)$ such that

$$
\varphi_{\sharp}\left(p_{i}\right)-\left(L_{n} \varphi\right)_{\sharp}\left(p_{i}\right) \equiv K x_{i n} .
$$

Combining this with (32) we obtain

$$
\alpha_{*}\left(p_{i}\right)-\left(L_{n} \varphi\right)_{\sharp}\left(p_{i}\right)=\left(\alpha_{*}\left(p_{i}\right)-\varphi_{\sharp}\left(p_{i}\right)\right)+\left(\varphi_{\sharp}\left(p_{i}\right)-\left(L_{n} \varphi\right)_{\sharp}\left(p_{i}\right)\right) \equiv K\left(-\gamma_{\sharp}\left(p_{i}\right)+x_{i n}\right) .
$$

Since $K_{0}(A)$ is a simple ordered group and $\alpha_{*}$ is order preserving, $\alpha_{*}[1]=[1]$, we have $\alpha_{*}\left(p_{i}\right)>0$ as $p_{i} \neq 0$. On the other hand $\left.\widehat{\alpha_{*}\left(p_{i}\right)}-L_{n} \widehat{\left(\varphi_{\sharp}\left(p_{i}\right)\right.}\right) \rightarrow \widehat{\alpha_{*}\left(p_{i}\right)}>0$, since $\widehat{L_{n}(x)} \rightarrow 0$ for all $x \in K_{0}(B)$ by part (c) of Definition 5.8. This shows that if $n$ is large enough then the image of $K\left(-\gamma_{\sharp}\left(p_{i}\right)+x_{i n}\right)$ in Aff $S\left(K_{0}(B)\right)$ is strictly positive. Since $K_{0}(B)$ is weakly unperforated, by Proposition 2.8(ii), we find projections $q_{i n} \in \operatorname{Proj}(B)$ such that $-\gamma_{\sharp}\left(p_{i}\right)+x_{i n}=\left[q_{i n}\right]$ for all $1 \leq i \leq r$. With $\Phi=L_{n} \varphi$ as above, we have $\alpha_{*}\left(p_{i}\right)-\Phi_{\sharp}\left(p_{i}\right)=K\left[q_{i n}\right]$. This proves $(30)$. Finally we note that since $\widehat{L_{n}\left(1_{B}\right)} \rightarrow 0$, by taking $n$ large enough, we obtain that $\left[\Phi\left(1_{A}\right)\right]<\left[1_{B}\right]$. Since $B$ has cancellation of projections, after conjugating $\Phi$ by a suitable partial isometry in $B \otimes \mathcal{K}$, we arrange that the image of $\Phi$ is contained in $B$ rather than just in $B \otimes \mathcal{K}$.

\section{Proof of MAin Results}

The following result is given in larger generality than needed in the paper, for the sake of future applications.

Theorem 6.1. Let $A, B$ be separable simple unital $C^{*}$-algebras with $\operatorname{TR}(A), T R(B) \leq 1$ and assume that $B$ is excisive (see Def. 5.8). Then for any $\alpha \in \operatorname{KK}_{n u c}(A, B)$ such that $\alpha_{*}\left(K_{0}(A)^{+}\right) \subset K_{0}(B)^{+}$and $\alpha_{*}\left[1_{A}\right]=\left[1_{B}\right]$ there is a ucp asymptotic morphism $\left(\Phi_{n}\right): A \rightarrow B$ consisting of nuclear maps $\Phi_{n}$ such that $\Phi_{n \sharp}(x) \equiv \alpha_{*}(x) \forall x \in \underline{\mathrm{K}}(A)$.

We are actually going to prove that the theorem is true whenever $A$ and $B$ satisfy the following conditions: (a) $A$ is an infinite dimensional separable simple unital $\mathrm{C}^{*}$-algebra with $K_{0}(A)$ a generalized dimension group and $A$ satisfies the conclusion of Proposition 4.4 (this also implies the quasidiagonality of $A$ ). (b) $B$ is an infinite dimensional separable simple stably finite unital excisive $C^{*}$-algebra with cancellation of projections and such that $K_{0}(B)$ is weakly unperforated.

Proof. Let $\alpha$ be as in the statement. We need to show that for any $\underline{K}$-triple $(\mathcal{P}, \mathcal{G}, \delta)$ there is a ucp map $\varphi: A \rightarrow B$ such that $\varphi$ is $(\mathcal{G}, \delta)$-multiplicative and $\varphi_{\sharp}(P)=\alpha_{*}[P]$ for all $P \in \mathcal{P}$. If $\mathcal{P}=\left\{P_{1}, \ldots, P_{\ell}\right\} \subset \operatorname{Proj}^{(M)}(A)$ for some integer $M$, we let $\mathcal{P}_{0}=\left\{p_{1}, \ldots, p_{\ell}\right\} \subset \operatorname{Proj}(A)$ be the image of $\mathcal{P}$ under the evaluation map $\operatorname{Proj}^{(M)}(A) \rightarrow \operatorname{Proj}(A)$. We have

$$
\left[P_{j}\right]-\left[p_{j}\right] \in K_{1}(A) \oplus \bigoplus_{m=2}^{M} K_{*}(A ; \mathbb{Z} / m)
$$

for all $j$. We may assume that $P_{\ell}=p_{\ell}=1_{A}$ and that $\left(\mathcal{P}_{0}, \mathcal{G}, \delta\right)$ is a $K_{0}$-triple. By Proposition 4.4 there is $R>0$ and there exists a $(\mathcal{G}, \delta)$-multiplicative ucp map $\psi: A \rightarrow M_{R}\left(A_{0}\right)$ such that

$$
R \rho\left[p_{j}\right]=(\psi)_{\sharp}\left(p_{j}\right), \quad \text { in } K_{0}\left(A_{0}\right), \quad \forall j 1 \leq j \leq \ell .
$$


If we set $K=R M !$ and replace $\psi$ by $\psi \otimes 1_{M !}: A \rightarrow M_{K}\left(A_{0}\right)$, then

$$
K \rho\left[p_{j}\right]=(\psi)_{\sharp}\left(p_{j}\right) \quad \text { in } K_{0}\left(A_{0}\right), \quad \forall j 1 \leq j \leq \ell .
$$

and from (34) $\psi$ has the additional property that

$$
\psi_{\sharp}\left(P_{j}\right)=\psi_{\sharp}\left(p_{j}\right) \quad \forall j 1 \leq j \leq \ell .
$$

Since $A_{0}$ is an AF algebra, after composing $\psi$ with a suitable conditional expectation we may assume that its image is contained in a finite dimensional $\mathrm{C}^{*}$-subalgebra $D$ of $M_{K}\left(A_{0}\right)$. Increasing $D$ to a larger a finite dimensional $\mathrm{C}^{*}$-subalgebra $C$ of $M_{K}\left(A_{0}\right)$ we may arrange that in addition to the property $\psi(A) \subset C$, there are projections $g_{j} \in \operatorname{Proj}(C)$ with

$$
\left[g_{j}\right]=\rho\left[p_{j}\right] \quad \text { in } K_{0}\left(A_{0}\right) \quad \text { and } \quad K\left[g_{j}\right]=\psi_{\sharp}\left(p_{j}\right) \quad \text { in } K_{0}(C), \quad 1 \leq j \leq \ell,
$$

Let $c_{s}, 1 \leq s \leq S$ be minimal projections in $C$, one for each subfactor of $C$. Then there are positive integers $k_{j s}$ such that

$$
\left[g_{j}\right]=\sum_{s=1}^{S} k_{j s}\left[c_{s}\right] \quad \text { in } K_{0}(C), \quad 1 \leq j \leq \ell .
$$

Since $\left[g_{j}\right]=\rho\left[p_{j}\right]$ we obtain:

$$
\rho\left[p_{j}\right]=\sum_{s=1}^{S} k_{j s}\left[c_{s}\right] \quad \text { in } K_{0}\left(A_{0}\right) .
$$

Let $d_{s} \in \operatorname{Proj}(A), 1 \leq s \leq S$ be such that

$$
\rho\left[d_{s}\right]=\left[c_{s}\right], \quad 1 \leq s \leq S .
$$

Then

$$
\left[p_{j}\right]-\sum_{s=1}^{S} k_{j s}\left[d_{s}\right] \in \operatorname{ker} \rho=\operatorname{Inf} K_{0}(A) .
$$

Let $\eta_{n}=\alpha-\Phi_{n \sharp}: \operatorname{Proj}^{(M)}(A) \rightarrow \underline{K}(B)$ where $\alpha$ and $\Phi_{n}$ are as in Proposition 5.9. By taking $n$ large enough, we arrange that $\Phi_{n}$ is $(\mathcal{G}, \delta)$-multiplicative and also from Proposition 5.9, (34) and (41) that

$$
\begin{gathered}
\eta_{n}\left(P_{j}\right)-\eta_{n}\left(p_{j}\right)=0, \quad 1 \leq j \leq \ell \\
\eta_{n}\left(p_{j}\right)=\sum_{s=1}^{S} k_{j s} \eta_{n}\left(d_{s}\right) \quad \text { in } K_{0}(B), \quad 1 \leq j \leq \ell .
\end{gathered}
$$

Also by construction of $\eta_{n}$ :

$$
\eta_{n}\left(d_{s}\right)=K\left[q_{s}\right] \quad \text { in } K_{0}(B), \quad 1 \leq s \leq S
$$

for some $q_{s} \in \operatorname{Proj}(B)$. Define $\theta_{*}: K_{0}(C) \rightarrow K_{0}(B)$ by

$$
\theta_{*}\left[c_{s}\right]=\left[q_{s}\right], \quad 1 \leq s \leq S
$$


and lift $\theta_{*}$ to a $*$-homomorphism $\theta: C \rightarrow B \otimes \mathcal{K}$. The role of $C$ is to enable us to define $\theta_{*}$ only on generators. This is something one cannot do at the level of $K_{0}\left(A_{0}\right)$. Using successively (37), (38), (45) (44) and (43) we have

$$
\begin{gathered}
(\theta \psi)_{\sharp}\left(p_{j}\right)=\theta_{*} \psi_{\sharp}\left(p_{j}\right)= \\
\left.\theta_{*}\left(K\left[g_{j}\right]_{K_{0}(C)}\right)=\theta_{*}\left(K \sum_{s=1}^{S} k_{j s}\left[c_{s}\right]_{K_{0}(C)}\right)\right)= \\
\sum_{s=1}^{S} k_{j s} K\left[q_{s}\right]_{K_{0}(B)}=\sum_{s=1}^{S} k_{j s} \eta_{n}\left[d_{s}\right]_{K_{0}(B)}=\eta_{n}\left(p_{j}\right)
\end{gathered}
$$

hence

$$
(\theta \psi)_{\sharp}\left(p_{j}\right)=\eta_{n}\left(p_{j}\right), \quad 1 \leq j \leq \ell .
$$

On the other hand we have

$$
(\theta \psi)_{\sharp}\left(P_{j}\right)=(\theta \psi)_{\sharp}\left(p_{j}\right), \quad 1 \leq j \leq \ell,
$$

by the definition of $p_{j}$ and (36). In combination with (42) this proves

$$
\eta_{n}\left(P_{j}\right)=(\theta \psi)_{\sharp}\left(P_{j}\right), \quad 1 \leq j \leq \ell .
$$

Equivalently

$$
\alpha_{*}\left(P_{j}\right)=\Phi_{n \sharp}\left(P_{j}\right)+(\theta \psi)_{\sharp}\left(P_{j}\right), \quad 1 \leq j \leq \ell .
$$

We conclude the proof by setting $\varphi=\Phi_{n} \oplus \theta \psi$. Let us note that a priori this map takes values in $B \otimes \mathcal{K}$. However since $\alpha_{*}\left[1_{A}\right]=\varphi_{\sharp}\left(1_{A}\right)=\left[1_{B}\right]$ and $B$ has cancellation of projections we can arrange that $\varphi\left(1_{A}\right)=1_{B}$ after conjugating $\varphi$ by a suitable partial isometry in $B \otimes \mathcal{K}$.

The class of model AH algebras is defined in Appendix A, Def. 7.2.

Proposition 6.2. Let $B$ be an infinite dimensional simple unital tracially $A F C^{*}$-algebra and let $C$ be a model $A H$ algebra. Then, for any $\alpha \in \operatorname{KK}(C, B)$ with $\alpha_{*}\left(K_{0}(C)^{+}\right) \subset K_{0}(B)^{+}$and $\alpha_{*}\left[1_{C}\right]=\left[1_{B}\right]$ there is a unital asymptotic morphism $\left(\Phi_{n}\right): C \rightarrow B$ such that $\Phi_{n \sharp}(x)=\alpha_{*}(x)$ for all $x \in \underline{\mathrm{K}}(C)$.

Proof. The result follows from Proposition 8.7 in Appendix B, since $B$ has cancellation of projections.

The following uniqueness result will allow us to construct $*$-homomorphisms out of approximate morphisms.

Theorem 6.3. [10] Let $A$ be a separable simple unital exact tracially AF $C^{*}$-algebra satisfying the UCT. For any finite subset $\mathcal{F} \subset A$ and any $\epsilon>0$, there exists a $\underline{K}$-triple $(\mathcal{P}, \mathcal{G}, \delta)$ with the following property. For any simple unital tracially $A F C^{*}$-algebra $B$ and any two nuclear ucp $(\mathcal{G}, \delta)$-multiplicative maps $\varphi, \psi: A \longrightarrow B$ satisfying $\varphi_{\sharp}(p)=\psi_{\sharp}(p)$ for all $p \in \mathcal{P}$, there exists a unitary $u \in U(B)$ such that $\left\|u \varphi(a) u^{*}-\psi(a)\right\|<\epsilon$ for all $a \in \mathcal{F}$.

In a recent paper [5], we showed that if $A$ is a separable $\mathrm{C}^{*}$-algebra satisfying the UCT, then two nuclear $*$-homomorphisms defined on $A$ are stably approximately unitarily equivalent if and only if they induce equal maps on $\underline{\mathrm{K}}(-)$. This result simplifies substantially the original proof of Theorem 6.3 by eliminating the discussion on algebraically compact groups.

We are ready now to prove the main result of the paper. 
Theorem 6.4. Let $A, B$ be separable simple unital tracially $A F C^{*}$-algebras. Suppose that $A$ is exact and satisfies the UCT. Then for any $\alpha \in \mathrm{KK}(A, B)$ with $\alpha_{*}\left(K_{0}(A)^{+}\right) \subset K_{0}(B)^{+}$and $\alpha_{*}\left[1_{A}\right]=\left[1_{B}\right]$ there is a nuclear unital $*$-homomorphism $\varphi: A \rightarrow B$ such that $\varphi_{*}(x)=\alpha_{*}(x)$ for all $x \in \underline{\mathrm{K}}(A)$. If $\psi: A \rightarrow B$ is another nuclear $*$-homomorphism with $\psi_{*}=\varphi_{*}: \underline{K}(A) \rightarrow$ $\underline{K}(B)$, then there is a sequence of unitaries $u_{n} \in B$ such that $\left\|\varphi(a)-u_{n} \psi(a) u_{n}^{*}\right\| \rightarrow 0$ for all $a \in A$.

One may note that in view of Theorem 2.1, Theorem 6.4 remains true if we start with an element $\underline{\alpha} \in \operatorname{Hom}_{\Lambda}(\underline{K}(A), \underline{K}(B))$ rather than with $\alpha \in \mathrm{KK}(A, B)$.

Proof. This is well-known (and easy) if $A$ is finite dimensional, so that we may assume that $A$ is infinite dimensional. Let $\alpha$ be as in the statement. It induces a map $\alpha_{*} \in$ $\operatorname{Hom}_{\Lambda}(\underline{K}(A), \underline{K}(B))$. We show first that $\alpha_{*}$ lifts to an asymptotic morphism. Let $C$ be a excisive tracially AF algebra ( $\mathrm{C}$ is also a model $\mathrm{AH}$ algebra) given by Theorem 7.6 such that $\left(K_{0}(A), K_{0}(A)^{+},\left[1_{A}\right]\right) \cong\left(K_{0}(C), K_{0}(C)^{+},\left[1_{C}\right]\right)$ and $K_{1}(A) \cong K_{1}(C)$. Since $A$ satisfies the UCT, we find $\beta \in \mathrm{KK}(A, C)$ inducing these isomorphisms. By Theorem 6.1 there is a ucp asymptotic morphism $\left(\Psi_{n}\right): A \rightarrow C$ lifting $\beta_{*}: \underline{\mathrm{K}}(A) \rightarrow \underline{\mathrm{K}}(C)$. On the other hand by Proposition 6.2. there is a ucp asymptotic morphism $\left(\Phi_{n}\right): C \rightarrow B$ lifting $\alpha_{*} \beta_{*}^{-1}$. Then $\left(\Gamma_{n}\right)=\left(\Phi_{r(n)} \Psi_{n}\right): A \rightarrow B$ will be a ucp asymptotic morphism lifting $\alpha_{*} \beta_{*}^{-1} \circ \beta_{*}=\alpha_{*}$, provided that $(r(n))$ is chosen appropriately. Each individual map $\Gamma_{n}$ is nuclear as it factors through $C$. Let $\left\{a_{1}, a_{2}, \ldots\right\}$ be a dense subset of $A$. Let $\mathcal{F}_{n}=\left\{a_{1}, \ldots, a_{n}\right\}$ and let $\epsilon_{n}=2^{-n}$.

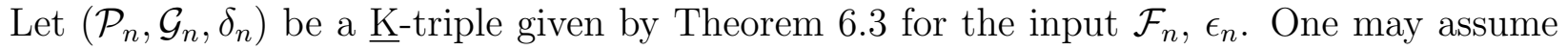
that $\mathcal{P}_{n} \subset \mathcal{P}_{n+1}, \mathcal{G}_{n} \subset \mathcal{G}_{n+1}$ and $\delta_{n}>\delta_{n+1}$. Passing to a subsequence of $\left(\Gamma_{n}\right)$ we may further assume that $\Gamma_{n}$ is $\left(\mathcal{G}_{n}, \delta_{n}\right)$-multiplicative and $\Gamma_{n \sharp}(p)=\alpha_{*}[p]$ for all $p \in \mathcal{P}_{n}$ and all $n$. By Theorem 6.3 there is a sequence of unitaries $\left(u_{n}\right)$ in $B$ such that $\left\|u_{n}^{*} \Gamma_{n}(a) u_{n}-\Gamma_{n+1}(a)\right\|<\epsilon_{n}$ for $a \in \mathcal{F}_{n}$. It follows that $\varphi_{n}=u_{1} u_{2} \ldots u_{n-1} \Gamma_{n} u_{n-1}^{*} \ldots u_{2}^{*} u_{1}^{*}$ is a ucp asymptotic morphism such that $\left(\varphi_{n}(a)\right)$ is a Cauchy sequence for all $a \in\left\{a_{1}, a_{2}, \ldots\right\}$, a dense subset of $A$. Thus $\varphi_{n}$ converges to a nuclear unital $*$-homomorphism $\varphi: A \rightarrow B$ with $\varphi_{*}=\alpha_{*}: \underline{K}(A) \rightarrow \underline{K}(B)$. The second part of the theorem follows from Theorem 6.3.

Theorem 6.5. (Lin) Let $A, B$ be separable simple unital nuclear tracially $A F C^{*}$-algebras satisfying the UCT. If $\left(K_{0}(A), K_{0}(A)^{+},\left[1_{A}\right]\right) \cong\left(K_{0}(B), K_{0}(B)^{+},\left[1_{B}\right]\right)$ and $K_{1}(A) \cong K_{1}(B)$, then $A$ is isomorphic to $B$.

Proof. This is very similar to the proof of the previous theorem. The case when $A$ (and hence $B)$ is finite dimensional is well known. Since $A$ satisfies the $\mathrm{UCT}$, there is $\alpha \in K K(A, B)$ inducing the isomorphism $\left(K_{0}(A), K_{0}(A)^{+},\left[1_{A}\right]\right) \cong\left(K_{0}(B), K_{0}(B)^{+},\left[1_{B}\right]\right)$. By Theorem 6.4 there are unital $*$-homomorphisms $\varphi: A \rightarrow B$ and $\psi: B \rightarrow A$ such that $\varphi_{*}=\alpha_{*}$ : $\underline{K}(A) \rightarrow \underline{K}(B)$ and $\psi_{*}=\alpha_{*}^{-1}$. Now $\varphi$ and $\psi$ are $*$-homomorphisms such that $\psi \circ \varphi$ is approximately unitarily equivalent to $i d_{A}$ and $\varphi \circ \psi$ is approximately unitarily equivalent to $i d_{B}$ by Theorem 6.4. One concludes that $A \cong B$ by Elliott's intertwining argument [17].

Corollary 6.6. If $D$ is a separable exact residually finite dimensional $C^{*}$-algebra satisfying the UCT, then $D$ is embeddable in an AF-algebra.

Proof. The idea of the proof goes back to [15] and is similar to the argument in [30]. One embeds first $D$ into a tracially AF algebra $A$ as in [7]. Since $A$ is an inductive limit of matrices over $D$ it satisfies the UCT and is exact. Let $A_{0}$ be the AF algebra introduced in Proposition 4.4. Then the map $\rho: K_{0}(A) \rightarrow K_{0}\left(A_{0}\right)$ lifts to a unital $*$-homomorphism $A \rightarrow A_{0}$. 
Another application is given in [9] where Theorem 6.4 is used in an essential manner to prove that if a nuclear separable $\mathrm{C}^{*}$-algebra $A$ has an exhausting sequence $\left(A_{n}\right)$ of $\mathrm{C}^{*}$ subalgebras such that each $A_{n}$ satisfies the UCT, then A satisfies the UCT. The subalgebras $A_{n}$ are necessarily exact but they may not be nuclear hence the relevance of the main result of the present paper.

\section{Appendix A}

Let $A$ be a separable simple unital tracially AF $\mathrm{C}^{*}$-algebra. By a result of [19] there is a simple "model" AH algebra $B$ with local spectra of dimension at most three and such that $B$ the same K-theory invariants as $A$. Here we refine this result (see Theorem 7.6) and show that in addition to the above properties $B$ can be taken to be a excisive tracially AF algebra (see Definition 5.8). The quoted result of [19] and its refinements given in [28] and here depend ultimately on a result of [14] which states that if $X$ and $Y$ are finite connected CW complexes with base points and $\operatorname{dim}(Y) \leq 3$ then every $*$-homomorphism $C_{0}(X) \rightarrow M_{K}\left(C_{0}(Y)\right)$ is homotopic to a $*$-homomorphism $C_{0}(X) \rightarrow M_{6}\left(C_{0}(Y)\right)$.

Let $W_{m}$ be a Moore space as defined in Section 2 and let $S W_{m}$ denote the suspension of $W_{m}$. A connected finite CW complex $X$ is called a model space if it is of the form

$$
X=Z_{0} \vee \cdots \vee Z_{r},
$$

where $r \geq 0, Z_{0}$ is the 3-dimensional simplex and

$$
Z_{i} \in\left\{S^{1}, S^{2}\right\} \cup\left\{W_{m}: m \geq 2\right\} \cup\left\{S W_{m}: m \geq 2\right\},
$$

for $1 \leq i \leq r$. We fix a base point $z_{i} \in Z_{i}$. The space $Z_{0} \vee \cdots \vee Z_{r}$ is the one point compactification of the disjoint union $\cup_{i=0}^{r}\left(Z_{i} \backslash z_{i}\right)$. Note that for any two finitely generated abelian groups $G_{1}, G_{2}$ there is a model space $X$ such that $\widetilde{K}^{0}(X)=G_{0}$ and $K^{1}(X)=G_{1}$. All model spaces have dimension three as they contain a copy of $Z_{0}$. The three dimensional spaces have nice cancellation properties for projections (vector bundles).

Remark 7.1. (a) If $X$ is a finite connected CW complex of dimension $\leq 3$, then any projection $p \in \operatorname{Proj}(C(X))$ of rank $\mathrm{k}$ is equivalent to $p_{0} \oplus 1_{k-1}$ where $p_{0}$ is a rank one projection. If $p, q \in \operatorname{Proj}(C(X))$ and $\operatorname{rank}(p)<\operatorname{rank}(q)$ then $p$ is equivalent to a subprojection of $q$. In particular $[p] \leq[q]$ in $K_{0}(C(X))$.

(b) If $p, q \in \operatorname{Proj}(C(X))$ and $[p]=[q]$ in $K_{0}(C(X))$, then $u p u^{*}=q$ for some unitary $u \in C(X)$

(c) $\operatorname{Inf} K_{0}(C(X))=\widetilde{K}^{0}(X)=\{[p]-[q]: p, q \in \operatorname{Proj}(C(X)), \operatorname{rank}(p)=\operatorname{rank}(q)=1\}$.

In what follows, $X$ and $Y$ will always denote model spaces and $P$ and $Q$ will be projections in $M_{N}\left(C(X)\right.$ and respectively $M_{N}(C(Y))$ for some $N \geq 1$. The $\mathrm{C}^{*}$-algebra $P M_{N}(C(X)) P$ will be denoted by $P(X)$ and $Q M_{N}(C(Y)) Q$ by $Q(Y)$. A $\mathrm{C}^{*}$-algebra of the form $P_{1}\left(X_{1}\right) \oplus$ $\cdots \oplus P_{n}\left(X_{n}\right)$ where $X_{i}$ are model spaces will be called a model homogeneous $\mathrm{C}^{*}$-algebra.

Definition 7.2. A C*-algebra isomorphic to the limit of an inductive system of model homogeneous $\mathrm{C}^{*}$-algebras with injective connecting maps will be called a model AH algebra.

Let $\gamma: P(X) \rightarrow Q(Y)$ be a $*$-homomorphism. The rank of $\gamma$ is defined by $\operatorname{rank}(\gamma)=$ $\operatorname{rank}(\gamma(P)) / \operatorname{rank}(P)$. If $d \geq 1$ we say that $\gamma$ is a type $d *$-homomorphism if there are unital *-homomorphisms $\gamma^{\prime}: P(X) \rightarrow Q^{\prime}(Y)$ and $\gamma^{\prime \prime}: P(X) \rightarrow Q^{\prime \prime}(Y)$ with orthogonal range and $\gamma=\gamma^{\prime}+\gamma^{\prime \prime}$ and such that the following two properties are satisfied. 
(i) $7 d \leq \operatorname{rank}\left(\gamma^{\prime}\right)<8 d, \quad \operatorname{rank}\left(\gamma^{\prime \prime}\right)=c d$, for some $c \geq 0$

(ii) $\gamma^{\prime \prime}$ admits a factorization

$$
P(X) \stackrel{\nu}{\rightarrow} F \stackrel{\iota}{\rightarrow} M_{d}(F) \stackrel{J}{\hookrightarrow} Q^{\prime \prime}(Y)
$$

where $F$ is a finite dimensional $C^{*}$-algebra, all the maps are unital $*$-homomorphisms and $\iota(b)=b \otimes 1_{d}$ for all $b \in F$.

More generally, a unital $*$-homomorphism

$$
\gamma: B=P_{1}\left(X_{1}\right) \oplus \cdots \oplus P_{n}\left(X_{n}\right) \rightarrow C=Q_{1}\left(Y_{1}\right) \oplus \cdots \oplus Q_{m}\left(Y_{m}\right)
$$

is called type $d$ if all its components $\gamma_{i j}: P_{i}\left(X_{i}\right) \rightarrow Q_{j}\left(Y_{j}\right)$ are type $d$. We have decompositions $\gamma_{i j}=\gamma_{i j}^{\prime}+\gamma_{i j}^{\prime \prime}$ as above and if $\gamma^{\prime}, \gamma^{\prime \prime}: B \rightarrow C$ are the $*$-homomorphisms with components $\left(\gamma_{i j}^{\prime}\right)$ and $\left(\gamma_{i j}^{\prime \prime}\right)$, then $\gamma=\gamma^{\prime}+\gamma^{\prime \prime}$. If $e=\gamma^{\prime}\left(1_{B}\right)$ then $e$ commutes with $\gamma(B)$, $\gamma^{\prime}=e \gamma e$ and $\gamma^{\prime \prime}$ admits a factorization

$$
B \stackrel{\nu}{\rightarrow} F \stackrel{\iota}{\rightarrow} M_{d}(F) \stackrel{\jmath}{\hookrightarrow}\left(1_{C}-e\right) C\left(1_{C}-e\right)
$$

where $F$ is a finite dimensional $C^{*}$-algebra, $\nu, \iota, \jmath$ are unital $*$-homomorphisms with $\jmath$ injective and $\iota(b)=b \otimes 1_{d}$ for all $b \in F$. This follows from the corresponding factorizations of the components $\gamma_{i j}^{\prime \prime}$. It will be convenient to suspend the notation for $j$ and write $\gamma^{\prime \prime}(b)=\nu(b) \otimes 1_{d}$ rather than $\gamma^{\prime \prime}(b)=\jmath\left(\nu(b) \otimes 1_{d}\right)$.

Remark 7.3. If the rank of each component $\gamma_{i j}$ is $\geq 8 n d$, then $n[e] \leq\left[1_{C}\right]$ in $K_{0}(C)$. Indeed, since the spaces $X_{i}, Y_{j}$ have dimension $\leq 3$, it suffices to verify that

$$
\operatorname{nrank}\left(\gamma_{i j}^{\prime}\left(P_{i}\right)\right)<\operatorname{rank}\left(\gamma_{i j}\left(P_{i}\right)\right)
$$

for each $i, j$. This is easily checked since $\operatorname{rank}\left(\gamma_{i j}\right) \geq 8 n d>n \operatorname{rank}\left(\gamma_{i j}^{\prime}\right)$.

Assume that $\alpha_{0}: K_{0}(P(X)) \rightarrow K_{0}(Q(Y))$ is a non-zero positive morphism of ordered groups. We have $K_{0}(P(X))=\widetilde{K}^{0}(X) \oplus \mathbb{Z}$ and

$$
K_{0}(P(X))^{+}=\left\{(x, r): x \in \widetilde{K}^{0}(X), r \in \mathbb{Z}, r>0\right\} \cup\{(0,0)\} .
$$

We can identify $\alpha_{0}$ with a matrix $\left(\begin{array}{cc}\alpha & \beta \\ 0 & k\end{array}\right)$ where $\alpha: \widetilde{K}^{0}(X) \rightarrow \widetilde{K}^{0}(Y), \beta: \mathbb{Z} \rightarrow \widetilde{K}^{0}(Y)$ and $k: \mathbb{Z} \rightarrow \mathbb{Z}, r \mapsto k r$ are group morphisms. The $(2,1)$ entry of this matrix is zero and $k \geq 1$ as a consequence of positivity of $\alpha_{0}$. The integer $k$ is called the rank of $\alpha_{0}$. Note that this definition is compatible with the definition of the rank of a $*$-homomorphism $\gamma: P(X) \rightarrow$ $Q(Y)$ in the sense that $\operatorname{rank}(\gamma)=\operatorname{rank}\left(K_{0}(\gamma)\right)$. More generally, if $\alpha_{0}: K_{0}(B) \rightarrow K_{0}(C)$ is a positive morphism with $B=P_{1}\left(X_{1}\right) \oplus \cdots \oplus P_{n}\left(X_{n}\right)$ and $C=Q_{1}\left(Y_{1}\right) \oplus \cdots \oplus Q_{m}\left(Y_{m}\right)$, then we define the component $\alpha_{0}^{i j}$ of $\alpha_{0}$ via the composition

$$
K_{0}\left(P_{i}\left(X_{i}\right)\right) \rightarrow K_{0}(B) \stackrel{\alpha_{0}}{\rightarrow} K_{0}(C) \rightarrow K_{0}\left(Q_{j}\left(Y_{j}\right)\right) .
$$

It is obvious that $\alpha_{0}^{i j} \geq 0$ if $\alpha \geq 0$. The rank matrix of $\alpha_{0}$ is defined to be the matrix $\left(k_{i j}\right)$ where $k_{i j}=\operatorname{rank}\left(\alpha_{0}^{i j}\right)$.

Let $\varphi: P(X) \rightarrow Q(Y)$ be a $*$-homomorphism and let $y \in Y$. The map $a \rightarrow \varphi(a)(y)$ is a finite dimensional representation of $P(X)$. It decomposes as a direct sum of irreducible representations given by points $x_{1}, \ldots, x_{m} \in X$. The set $\left\{x_{1}, \ldots, x_{m}\right\}$ is called the spectrum of $\varphi$ at $y$ and is denoted by $\operatorname{SP}\left(\varphi_{y}\right)$. This notion extends in the obvious way to $*$-homomorphisms $\varphi: A \rightarrow B$ between homogeneous $\mathrm{C}^{*}$-algebras $A, B$ [19]. Assume that the spectra of $A$ and $B$ are compact metric spaces. We say that a $*$-homomorphism $\varphi: A \rightarrow B$ is $\epsilon$-full if for 
any $y$ in the spectrum of $B, \operatorname{SP}\left(\varphi_{y}\right)$ is $\epsilon$-dense in the spectrum of $A$, i.e. $\operatorname{dist}\left(x, \operatorname{SP}\left(\varphi_{y}\right)\right)<\epsilon$ for all $x$ in the spectrum of $A$. The interest in notion this comes from a characterization of simple AH algebras given in [13]. Specifically, if $\left(A_{n}, \gamma_{n}\right)$ is an inductive system of homogeneous $\mathrm{C}^{*}$-algebras with injective connecting maps, then $\lim \left(A_{n}, \gamma_{n}\right)$ is simple if and only if

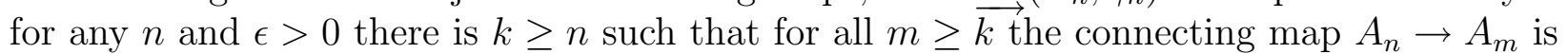
$\epsilon$-full. The proof of the following easy lemma is left to the reader. It is a consequence of the semicontinuity properties of the map $y \mapsto \operatorname{SP}\left(\varphi_{y}\right)$.

Lemma 7.4. Let $A,\left(A_{i}\right)_{i=1}^{n}$ be unital homogeneous $C^{*}$-algebras and let $\left(\varphi_{i}\right)_{i=1}^{n}$ be injective *-homomorphisms with $\varphi_{i}: A_{i} \rightarrow A$. For any $\epsilon>0$ there is $\delta>0$ such that for any unital homogeneous $C^{*}$-algebra $B$ and any $\delta$-full $*$-homomorphism $\psi: A \rightarrow B, \psi \varphi_{i}$ is $\epsilon$-full for all $1 \leq i \leq n$.

Theorem 7.5. Let $B$ be a model homogeneous $C^{*}$-algebra, let $\delta>0$ and let $d \geq 1$ be an integer. There is $m \geq 1$ such that for any model homogeneous $C^{*}$-algebra $C$ and any two morphisms $\alpha_{i}: K_{i}(B) \rightarrow K_{i}(C), i=0,1$ with $\alpha_{0}$ positive, $\alpha_{0}\left[1_{B}\right]=\left[1_{C}\right]$ and all the entries of the rank matrix of $\alpha_{0}$ greater than $8 \mathrm{md}$, there is a unital $*$-homomorphism $\gamma: B \rightarrow C$ with $K_{i}(\gamma)=\alpha_{i}, i=0,1$ such that $\gamma$ is type $d$ and $\delta$-full.

Proof. Since $\alpha_{0}^{i j}$ is positive and has rank at least 1, there is a subprojection $Q_{j i}$ of $Q_{j}$ such that $\alpha_{0}^{i j}\left[P_{i}\right]=\left[Q_{j i}\right]$. Since $K_{0}\left(Q_{j i}\left(Y_{j}\right)\right) \cong K_{0}\left(Q_{j}\left(Y_{j}\right)\right)$ as ordered groups, the proof of the theorem reduces to the case when both $B$ and $C$ have just one summand, say $B=P(X)$ and $C=Q(Y)$. Assume that $B, \delta$ and $d$ are given and let $\alpha_{i}$ be as in the statement of the theorem. Choose $m$ such that there are $m-1$ points $x_{1}, \ldots, x_{m-1}$ forming a $\delta$-dense subset of $X$. Since $\operatorname{rank}\left(\alpha_{0}\right)=k \geq 8 m d \geq 8 d$, we find integers $r, c \geq 0$ such that

$$
k=r+c d, \quad 7 d \leq r<8 d .
$$

As above, we identify $\alpha_{0}$ with a matrix and decompose it as follows.

$$
\alpha_{0}=\left(\begin{array}{cc}
\alpha & \beta \\
0 & k
\end{array}\right)=\left(\begin{array}{cc}
\alpha & 0 \\
0 & 6
\end{array}\right)+\left(\begin{array}{cc}
0 & \beta \\
0 & r-6
\end{array}\right)+\left(\begin{array}{cc}
0 & 0 \\
0 & c d
\end{array}\right)
$$

We are going to lift these matrices to $*$-homomorphisms. First we claim that there is a unital $*$-homomorphism $\varphi_{1}: C(X) \rightarrow M_{6}(C(Y))$ such that $K_{0}\left(\varphi_{1}\right)=\left(\begin{array}{ll}\alpha & 0 \\ 0 & 6\end{array}\right)$ and $K_{1}\left(\varphi_{1}\right)=$ $\alpha_{1}$. Note that it suffices to find a $*$-homomorphism $C_{0}(X) \rightarrow M_{6}\left(C_{0}(Y)\right)$ which induces $\alpha$ on $K_{0}$ and $\alpha_{1}$ on $K_{1}$ and let $\varphi_{1}$ be its unitalization. Let $k k(Y, X)$ denote the connective KK-theory of [14]. Assume that $X$ and $Y$ are connected finite CW complexes of dimension $\leq 3$. Then the map

$$
\left[C_{0}(X), M_{6}\left(C_{0}(Y)\right)\right] \rightarrow k k(Y, X)
$$

is a bijection by [14, Corollary 6.4.4]. Extending a result of [14] Elliott and Gong [19] have shown that the natural map

$$
k k(Y, X) \rightarrow K K\left(C_{0}(X), C_{0}(Y)\right)
$$

is a bijection if $H^{3}(X, \mathbb{Z})$ and $H^{3}(Y, \mathbb{Z})$ are torsion groups. Since the map

$$
K K\left(C_{0}(X), C_{0}(Y)\right) \rightarrow \operatorname{Hom}\left(K_{*}\left(C_{0}(X)\right), K_{*}\left(C_{0}(Y)\right)\right)
$$

is surjective by the UCT, the claim follows. Since all model spaces have a quotient homeomorphic to the 3-dimensional simplex, there is a null-homotopic continuous and surjective map $\pi: Y \rightarrow X$. Let $E$ be a projection in $M_{N}(C(Y))$ whose $K_{0}$-class is equal to $(\beta(1), r-6)$. 
Then $\varphi_{2}: C(X) \rightarrow E(Y), \varphi_{2}(f)(y)=f(\pi(y)) E(y)$ is a unital *-monomorphism with $K_{0}\left(\varphi_{2}\right)=\left(\begin{array}{cc}0 & \beta \\ 0 & r-6\end{array}\right)$ and $K_{1}\left(\varphi_{2}\right)=0$. Since $c d=k-r \geq 8 m d-8 d \geq m-1$, there is a $\delta$-dense subset $\left\{x_{1}, \ldots, x_{c d}\right\}$ of $X$. Define $\varphi_{3}: C(X) \rightarrow M_{c d}(\mathbb{C}) \subset M_{c d}(C(Y))$ by

$$
\varphi_{3}(f)(y)=\operatorname{diag}\left(f\left(x_{1}\right), \ldots, f\left(x_{c d}\right)\right), \quad y \in Y .
$$

Then $K_{0}\left(\varphi_{3}\right)=\left(\begin{array}{cc}0 & 0 \\ 0 & c d\end{array}\right), K_{1}\left(\varphi_{3}\right)=0$ and $\varphi_{3}$ is $\delta$-full. Set $\varphi=\varphi_{1} \oplus \varphi_{2} \oplus \varphi_{3}$ and let $N$ be large enough such that $P \in M_{N}(C(X))$. Then the restriction of $\widetilde{\varphi}=i d_{N} \otimes \varphi$ to $P(X)$ gives is a unital $*$-homomorphism $P(X) \rightarrow \widetilde{\varphi}(P)(Y)$ which implements $\alpha_{i}, i=0,1$. Since $\widetilde{\varphi}(P)$ is unitarily equivalent to $Q$ (as they have the same K-theory class), we conclude the proof by conjugating the restriction of $\widetilde{\varphi}$ to $P(X)$ by a suitable unitary. It is clear that this map is $\delta$-full since the direct summand $\varphi_{3}$ of $\varphi$ has this property.

The following result is a refinement of a result of Elliott and Gong [19] due to Lin [28].

Theorem 7.6. Let $\left(G, G^{+}, U\right)$ be a countable weakly unperforated simple scaled ordered group with the Riesz interpolation property and such that $G / G_{\text {tor }}$ is noncyclic. Let $K$ be a countable abelian group. There is an infinite dimensional separable simple unital model AH algebra $B$ such that $\left(K_{0}(B), K_{0}(B)^{+},\left[1_{B}\right]\right) \cong\left(G, G^{+}, U\right)$ and $K_{1}(B) \cong K$. Moreover we can arrange that $B$ is a excisive tracially $A F$ algebra.

Proof. We are going to show that for any sequence $\left(d_{n}\right)$ with $d_{n} \geq 1$ we find $B=\underline{\lim }\left(B_{n}, \gamma_{n}\right)$ where $B_{n}$ are model homogeneous $\mathrm{C}^{*}$-algebras and $\gamma_{n}$ are unital $*$-monomorphisms of type $d_{n}$ with the each entry of the rank matrix of $\gamma_{n}$ greater than $8 n d_{n}$. Moreover we will arrange that $\operatorname{Inf} K_{0}(B) \cong \lim _{\longrightarrow}\left(\operatorname{Inf} K_{0}\left(B_{n}\right), \gamma_{n *}\right)$.

By Theorem $2.6 \vec{G}$ has the strict ordering induced from the map $\rho: G \rightarrow \operatorname{Aff} S(G)$. The image $R=\rho(G)$ of the map $\rho$ is uniformly dense in $\operatorname{Aff} S(G)$ and $\left(R, R^{+}, u\right)$ is a scaled dimension group, where $u=\rho(U)$. Consider the extension

$$
0 \rightarrow H \rightarrow G \stackrel{\rho}{\rightarrow} R \rightarrow 0
$$

where $H=\operatorname{ker} \rho=\operatorname{Inf}(G)$. We find a system $\left(R_{n}, R_{n}^{+}, u_{n}\right)=\left(\mathbb{Z}^{m(n)}, \mathbb{Z}^{m(n)+}, u_{n}\right)$ of ordered scaled groups with order and scale preserving connecting maps $\kappa_{n}$ and maps $\kappa_{\infty, n}: R_{n} \rightarrow R$ satisfying $\kappa_{\infty, n+1} \kappa_{n}=\kappa_{\infty, n}$ with the property that the induced map $\kappa: \lim \left(R_{n}, \kappa_{n}\right) \rightarrow R$ is an isomorphism of ordered scaled groups. This is possible since $R$ is a dimension group. Moreover, since $R$ is simple, one can arrange that each entry of the matrix of $\kappa_{n}$ is an integer greater than $8 n d_{n}$.

Next we construct pullbacks

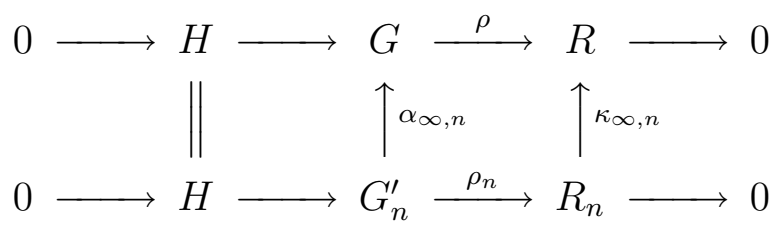


and observe that there are maps $\alpha_{n}: G_{n}^{\prime} \rightarrow G_{n+1}^{\prime}$ (induced canonically by the maps $\kappa_{n}$ ) with $\alpha_{\infty, n+1} \alpha_{n}=\alpha_{\infty, n}$ and such that we have commutative diagrams

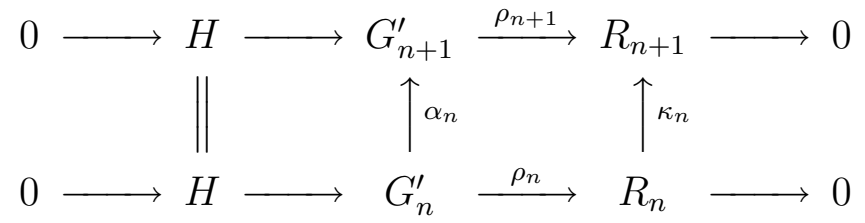

It is clear that the maps $\alpha_{\infty, n}$ induce an isomorphism $\alpha: \underline{\lim }\left(G_{n}^{\prime}, \alpha_{n}\right) \rightarrow G$.

The next step is to replace $H$ and $G_{n}^{\prime}$ by finitely generated subgroups. Since $G$ is countable and $R_{n}$ is finitely generated, we construct inductively finitely generated subgroups $G_{n}$ of $G_{n}^{\prime}$ such that $\rho_{n}\left(G_{n}\right)=R_{n}, \alpha_{n}\left(G_{n}\right) \subset G_{n+1}$ and $\cup_{n=1}^{\infty} \alpha_{\infty, n}\left(G_{n}\right)=G$. Define $H_{n}=\left\{x \in G_{n}\right.$ : $\left.\rho_{n}(x)=0\right\}$. By restricting the above diagram to suitable subgroups we obtain a commutative diagram

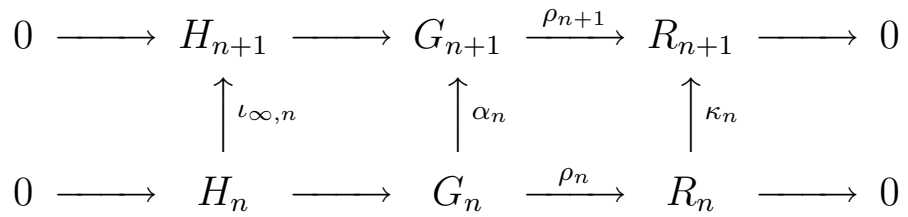

By construction we have an isomorphism $\alpha: \underline{\lim }\left(G_{n}, \alpha_{n}\right) \rightarrow G$ which induces an isomorphism $\underset{\lim }{\longrightarrow}\left(H_{n}, \iota_{n}\right) \rightarrow H$. After suitable reindexing, we may arrange that there is $U_{1} \in G_{1}$ such that $\alpha_{\infty, 1}\left(U_{1}\right)=U$ and $\rho_{1}\left(U_{1}\right)=u_{1}$. Then we define $U_{n+1}=\alpha_{n}\left(U_{n}\right)$. We are going to put an ordering on $G_{n}$ such that $U_{n}$ will be an order unit, $\alpha_{n}$ are positive and

$$
\left(G, G^{+}, U\right)=\lim _{\longrightarrow}\left(G_{n}, G_{n}^{+}, U_{n}\right) .
$$

Using sections $\mathbb{Z}^{m(n)} \rightarrow G_{n}$ we identify $G_{n}$ with $H_{n} \oplus \mathbb{Z}^{m(n)}$. Define $G_{n}^{(i)}=H_{n}^{(i)} \oplus \mathbb{Z}$ where $H_{n}^{(1)}=H_{n}$ and $H_{n}^{(i)}=0$ if $1<i \leq m(n)$. Let $\left(G_{n}^{(i)}\right)^{+}=\left\{(h, r) \in H_{n}^{(i)} \oplus \mathbb{Z}: r>0\right\} \cup\{(0,0)\}$ and define $G_{n}^{+}=\oplus_{i}\left(G_{n}^{(i)}\right)^{+}$. The connecting maps $\alpha_{n}$ are positive since all the matrix entries of $\kappa_{n}$ are strictly positive. Moreover each component of $U_{n}$ is strictly positive since $u_{n}$ has the same property. Since we already know that $G=\underset{\lim }{\longrightarrow}\left(G_{n}, \alpha_{n}\right)$, in order to verify (50), it suffices to show that for any nonzero $g \in G^{+}$there is $n$ and $g_{n} \in G_{n}^{+}$such that $\alpha_{\infty, n}\left(g_{n}\right)=g$. Let $g_{n} \in G_{n}$ be a lifting of $g$. Then $\rho_{n}\left(g_{n}\right)$ is a lifting of $\rho(g)$. Hence, after increasing $n$, we may assume that $\rho_{n}\left(g_{n}\right) \in \mathbb{Z}^{m(n)}$ has all the components strictly positive. But this clearly implies that $g_{n} \in G_{n}^{+}$.

Write $K$ as the inductive limit of a system $\left(K_{n}, \alpha_{n}^{1}\right)$ of finitely generated abelian groups. The system $\left(B_{n}, \gamma_{n}\right)$ is constructed inductively. In a first stage, this goes as follows. For each $n$ and each $i, 1 \leq i \leq m(n)$ let $X_{n}^{(i)}$ be a model space with $\widetilde{K}^{0}\left(X_{n}^{(i)}\right)=H_{n}^{(i)}$ and $K^{1}\left(X_{n}^{(i)}\right)=K_{n}^{(i)}$ where $K_{n}^{(1)}=K_{n}$ and $K_{n}^{(i)}=0$ for $i>1$. Let $P_{n}^{(i)}$ be projections in $M_{N}\left(C\left(X_{n}^{(i)}\right)\right)$ with $\left[P_{n}^{(i)}\right]=U_{n}^{(i)}$ where $U_{n}^{(i)}$ are the components of $U_{n}$ in $G_{n}^{(i)}=K_{0}\left(C\left(X_{n}^{(i)}\right)\right)$.

If we define $B_{n}=\oplus_{i=1}^{m(n)} P_{n}^{(i)}\left(X_{n}^{(i)}\right)$ then $\left(K_{0}\left(B_{n}\right), K_{0}\left(B_{n}\right)^{+},\left[1_{B_{n}}\right]\right)=\left(G_{n}, G_{n}^{+}, U_{n}\right)$ and $\operatorname{Inf} K_{0}\left(B_{n}\right)=H_{n}$. The rank matrix of $\alpha_{n}: K_{0}\left(B_{n}\right) \rightarrow K_{0}\left(B_{n+1}\right)$ coincides with the matrix of the map $\kappa_{n}$, whose entries are all positive and $\geq 8 n d_{n}$. By Theorem $7.5, \alpha_{n}$ lifts to a type $d_{n}$ *-monomorphism $\gamma_{n}: B_{n} \rightarrow B_{n+1}$ with $K_{i}\left(\gamma_{n}\right)=\alpha_{n}^{i}$. The above construction of the inductive system $\left(B_{n}, \gamma_{n}\right)$ has all the desired properties, except that we do not know that its inductive limit $\mathrm{C}^{*}$-algebra $B$ is simple. In order to insure simplicity of $B$ we refine the construction as follows. Let $\epsilon_{n}=1 / n$ and choose $B_{1}$ as above. Assume that we constructed $B_{1}, \ldots, B_{n}$ and type $d_{i}$ *-monomorphism $\gamma_{i}: B_{i} \rightarrow B_{i+1}$ which lift $\alpha_{i}$ for $1 \leq i \leq n-1$ and 
such that all the composite maps $B_{i} \rightarrow B_{n}$ are $\epsilon_{n}$-full. Let $\delta$ be given by Lemma 7.4 for the input data consisting of $\epsilon_{n+1}$, the algebras $B_{1}, \ldots, B_{n}$ and the maps $B_{i} \rightarrow B_{n}$. We may assume that $0<\delta<\epsilon_{n+1}$. Let $m$ be given by Theorem 7.5. By telescoping the inductive systems constructed in the first part of the proof we may assume that the rank matrix of $\alpha_{n}: K_{0}\left(B_{n}\right) \rightarrow K_{0}\left(B_{n+1}\right)$ (which coincides with the matrix of the map $\kappa_{n}$ ) has all entries $\geq 8 \mathrm{mnd}_{n}$. By Theorem 7.5, in addition to the properties above, we can arrange that $\gamma_{n}$ is $\delta$-full. Thus all that connecting maps $B_{i} \rightarrow B_{n+1}(i \leq n+1)$ are $\epsilon_{n+1}$-full by Lemma 7.4. The simplicity of $B$ follows now from a result of [13] since $\left(\epsilon_{n}\right)$ converges to zero. We verify in the next proposition that $B$ is tracially $\mathrm{AF}$ and excisive.

Proposition 7.7. Let $\left(B_{n}, \gamma_{n}\right)$ be an inductive system of model homogeneous $C^{*}$-algebras where $\gamma_{n}$ are unital $*$-monomorphisms of type $n$ ! with the entries of the rank matrix of $\gamma_{n}$ greater than $8 n n !$. Assume that $B=\lim _{\longrightarrow}\left(B_{n}, \gamma_{n}\right)$ is simple and that

$$
\operatorname{Inf} K_{0}(B) \cong \lim _{\longrightarrow}\left(\operatorname{Inf} K_{0}\left(B_{n}\right), \gamma_{n *}\right) .
$$

Then $B$ is a excisive tracially $A F C^{*}$-algebra.

Proof. Let $d_{n}=n$ !. We are going to prove that there is a sequence $\left(e_{n}\right)$ of projections in $B$ and there exists a ucp asymptotic morphism $\left(\mu_{n}\right)$, where $\mu_{n}: B \rightarrow F_{n}$ with $F_{n}$ finite dimensional $\mathrm{C}^{*}$-algebras, and there are unital embeddings $M_{d_{n}}\left(F_{n}\right) \hookrightarrow\left(1-e_{n}\right) B\left(1-e_{n}\right)$ such that the following six conditions satisfied.

(1) $\lim _{n \rightarrow \infty}\left[b, e_{n}\right]=0$ for all $b \in B$.

(2) $\lim _{n \rightarrow \infty}\left\|b-e_{n} b e_{n}-\mu_{n}(b) \otimes 1_{d_{n}}\right\|=0$ for all $b \in B$.

(3) $n\left[e_{n}\right] \leq\left[1_{B}\right]$ in $K_{0}(B)$

(4) If $L_{n}: B \rightarrow B$ is defined by $L_{n}(b)=e_{n} b e_{n}$, then $L_{n, \sharp}(x) \equiv x$ for all $x \in \operatorname{Inf} \underline{K}(B)$.

(5) For any $x \in K_{0}(B), \lim _{n \rightarrow \infty} \widehat{L_{n \sharp}(x)}=0$ and for any $K \in \mathbb{Z}$ there is a sequence $\left(x_{n}\right)$ in $K_{0}(B)$ such that $x-L_{n, \sharp}(x) \equiv K x_{n}$.

In particular this will prove $B$ is a excisive tracially AF algebra.

Since $\gamma_{n}: B_{n} \rightarrow B_{n+1}$ is type $d_{n}$, we have a decomposition $\gamma_{n}=\gamma_{n}^{\prime} \oplus \gamma_{n}^{\prime \prime}$ and a projection $e_{n}=\gamma_{n}^{\prime}\left(1_{B_{n}}\right)$ which commutes with $\gamma_{n}\left(B_{n}\right)$. Moreover, $\gamma_{n}^{\prime \prime}$ admits a factorization

$$
B_{n} \stackrel{\nu_{n}}{\rightarrow} F_{n} \stackrel{\iota_{n}}{\rightarrow} M_{d_{n}}\left(F_{n}\right) \hookrightarrow\left(1-e_{n}\right) B_{n+1}\left(1-e_{n}\right),
$$

with $\iota_{n}(b)=b \otimes 1_{d_{n}}$, so that $\gamma_{n}^{\prime \prime}=\nu_{n} \otimes 1_{d_{n}}$. Since $\gamma_{n}$ is injective we may regard $B_{n}$ as forming an increasing sequence of subalgebras of $B$ whose union is dense in $B$. We may also identify $e_{n}$ with its image in $B$. Let $\mu_{n}: B \rightarrow F_{n}$ be a ucp extension of $\nu_{n}$ to $B$. With these identifications, the identity $\gamma_{n}(b)=\gamma_{n}^{\prime}(b)+\gamma_{n}^{\prime \prime}(b)$ becomes

$$
b=e_{n} b e_{n}+\nu_{n}(b) \otimes 1_{d_{n}}=e_{n} b e_{n}+\mu_{n}(b) \otimes 1_{d_{n}}, \forall b \in B_{n} .
$$

Let us verify the conclusions of the proposition. To prove (1) we observe that $e_{n}$ commutes with $B_{n}$ and the union of the $B_{n}$ is dense in $B$. Arguing similarly, (2) follows from (51). We noted in Remark 7.3 that $n\left[e_{n}\right] \leq\left[1_{B_{n+1}}\right]$, and this implies $(3)$. We are ready to prove that $B$ is tracially AF. In view of properties (1) and (2), since $B$ has cancellation of projections, it suffices to verify that for any nonzero projection $q \in B$ there is $m$ such that $\left[e_{n}\right]<[q]$ for $n>m$. Since $B$ is simple, there is $m$ such that $\left[1_{B}\right] \leq m[q]$. Thus if $n>m$ then $n\left[e_{n}\right] \leq\left[1_{B}\right] \leq m[q]<n[q]$. Since the $K_{0}$ group of a model AH algebra is weakly unperforated it follows that $\left[e_{n}\right]<[q]$. 
Next we verify (4). As a consequence of (2) we have

$$
x \equiv L_{n \sharp}(x)+n ! \mu_{n \sharp}(x), \quad \forall x \in \underline{K}(B) .
$$

If $x \in K_{*}(B ; \mathbb{Z} / m)$, then $m x=0$ and the conclusion follows from (52). Let $x \in \operatorname{Inf} K_{0}(B) \oplus$ $K_{1}(B)$. We have $K_{*}(B)=\lim _{\longrightarrow}\left(K_{*}\left(B_{n}\right), \gamma_{n *}\right)$ by continuity of K-theory and $\operatorname{Inf} K_{0}(B)=$

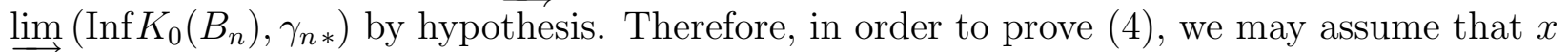

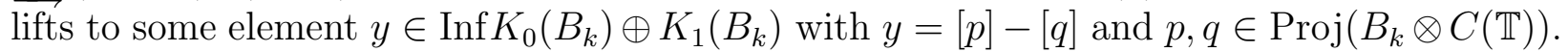
If $n \geq k$, then $[p]-[q] \in \operatorname{Inf} K_{0}\left(B_{n}\right) \oplus K_{1}\left(B_{n}\right)$. Since $\nu_{n}$ is a $*$-homomorphism with range contained in $F_{n}$, we have that $\nu_{n}$ vanishes on $\operatorname{Inf} K_{0}\left(B_{n}\right) \oplus K_{1}\left(B_{n}\right)$, because both $\operatorname{Inf} K_{0}(-)$ and $K_{1}(-)$ are trivial for finite dimensional $\mathrm{C}^{*}$-algebras. Thus $\nu_{n *}([p]-[q])=0$. From $(52)$ we have

$x \equiv L_{n \sharp}(x)+d_{n} \mu_{n \sharp}(x) \equiv L_{n \sharp}(x)+d_{n}\left(\mu_{n \sharp}(p)-\mu_{n \sharp}(q)\right) \equiv L_{n \sharp}(x)+d_{n} \nu_{n *}([p]-[q]) \equiv L_{n \sharp}(x)$ hence $L_{n \sharp}(x) \equiv x$. The first part of (5) follows since $\widehat{L_{n}(1)}=\widehat{e}_{n} \leq 1 / n$ by (3). The second part is an obvious consequence of (52).

\section{Appendix B}

The goal of this section is to give a short proof of Prop. 8.7. Let $A$ be a model AH algebra and let $B$ be a tracially AF algebra. We address the question of which elements of $K K(A, B)$ lift to approximate morphisms from $A$ to $B \otimes \mathcal{K}$. The all important case when $A=C_{0}(X)$ was already considered in Corollary 5.7.

Definition 8.1. Let $A, B$ be separable $\mathrm{C}^{*}$-algebras. We say that $A$ is $K$-related to $B$, written $A \stackrel{K}{\rightsquigarrow} B$, if for any $\alpha_{*} \in \operatorname{Hom}_{\Lambda}(\underline{K}(A), \underline{K}(B))$ with $\alpha_{*}\left(K_{0}(A)^{+}\right) \subset K_{0}(B)^{+}$and for any $\underline{K}$-triple $(\mathcal{P}, \mathcal{G}, \delta)$ there is a ccp nuclear map $\varphi: A \rightarrow B \otimes \mathcal{K}$ such that $\varphi$ is $(\mathcal{G}, \delta)$ multiplicative and $\varphi_{\sharp}(P)=\alpha_{*}[P]$ for all $P \in \mathcal{P}$. Equivalently, there is a ccp asymptotic morphism $\left(\varphi_{n}\right): A \rightarrow B \otimes \mathcal{K}$ with each individual map $\varphi_{n}$ nuclear such that $\varphi_{n \sharp}(x) \equiv \alpha_{*}(x)$ for all $x \in \underline{K}(A)$. We say that $\left(\varphi_{n}\right)$ is a lifting of $\alpha_{*}$.

Note that if $A$ and $B$ are as in Proposition 6.1 and $A$ satisfies the UCT, then $A \stackrel{K}{\rightsquigarrow} B$. Indeed if $A$ satisfies the UCT, then $\alpha_{*}$ lifts to a $\mathrm{KK}$-element $\alpha \in \mathrm{KK}(A, B)$.

Lemma 8.2. (a) If $A_{1} \stackrel{K}{\rightsquigarrow} B$ and $A_{2} \stackrel{K}{\rightsquigarrow} B$, then $A_{1} \oplus A_{2} \stackrel{K}{\rightsquigarrow} B$.

(b) Assume that $A$ is unital and $p \in M_{N}(A)$ is a full projection. If $A \stackrel{K}{\rightsquigarrow} B$, then $p M_{N}(A) p \stackrel{K}{\rightsquigarrow} B$.

(c) Let $\left(A_{i}\right)$ be an exhausting sequence of $C^{*}$-subalgebras of a separable $C^{*}$-algebra $A$. If $A_{i} \stackrel{K}{\rightsquigarrow} B$ for all $i$, then $A \stackrel{K}{\rightsquigarrow} B$.

Proof. (a) and (b) are easy exercises. For (b) one uses the stable isomorphism between $A$ and $M_{N}(A)$. For (c) one proceeds as follows. Let $\alpha_{*}$ is as in Definition 8.1. By assumption, for each $i$, there is a ccp asymptotic morphism $\left(\varphi_{n}^{(i)}\right): A_{i} \rightarrow B \otimes \mathcal{K}$ which lifts the composite map

$$
\underline{\mathrm{K}}\left(A_{i}\right) \rightarrow \underline{\mathrm{K}}(A) \stackrel{\alpha_{*}}{\rightarrow} \underline{\mathrm{K}}(B) .
$$

Moreover each individual map $\varphi_{n}^{(i)}$ is nuclear. Thus we may arrange that $\varphi_{n}^{(i)}$ factors through a finite dimensional $\mathrm{C}^{*}$-algebra and in fact that $\varphi_{n}^{(i)}$ extends to a ccp map $A \rightarrow B \otimes \mathcal{K}$ denoted in the same way. Finally, it is not hard to show that for a suitable choice of $\left(i_{n}\right)$, the sequence $\left(\varphi_{n}^{\left(i_{n}\right)}\right): A \rightarrow B \otimes \mathcal{K}$ defines a ccp asymptotic morphism which lifts $\alpha_{*}$. 
Lemma 8.3. Let $A$ be a separable simple unital tracially $A F C^{*}$-algebra. For any $x \in$ $\operatorname{Inf} K_{0}(A)$, there are projections $p_{n}, q_{n}$ in $A$ such that $x=\left[p_{n}\right]-\left[q_{n}\right]$ and $\widehat{p}_{n} \rightarrow 0, \widehat{q}_{n} \rightarrow 0$.

Proof. We may assume that $A$ is infinite dimensional. By Theorem 2.8 (i) there is a sequence of projections $\left(p_{n}\right)$ in $A$ with $0<\widehat{p}_{n}<1$ and $\widehat{p}_{n} \rightarrow 0$. Since $\widehat{p}_{n}-\widehat{x}=\widehat{p}_{n}$, by part (ii) of the same theorem we have that $0<\left[p_{n}\right]-x<\left[1_{A}\right]$. Therefore, since $A$ has cancellation of projections, there is a sequence of projections $\left(q_{n}\right)$ in $A$ with $\left[q_{n}\right]=\left[p_{n}\right]-x$. We have that $\widehat{q}_{n} \rightarrow 0$ since $\widehat{q}_{n}=\widehat{p}_{n}$.

Proposition 8.4. Let $B$ be an infinite dimensional separable simple unital tracially $A F C^{*}$ algebra and let $d_{n}=n$ !. Then there is a sequence $\left(e_{n}\right)$ of projections in $B$ and there exist a ucp asymptotic morphism $\mu_{n}: B \rightarrow F_{n}$ with $F_{n}$ finite dimensional $C^{*}$-algebras and unital embeddings $M_{d_{n}}\left(F_{n}\right) \hookrightarrow\left(1-e_{n}\right) B\left(1-e_{n}\right)$ such that

(1) $\lim _{n \rightarrow \infty}\left[b, e_{n}\right]=0$ for all $b \in B$.

(2) $\lim _{n \rightarrow \infty}\left\|b-e_{n} b e_{n}-\mu_{n}(b) \otimes 1_{d_{n}}\right\|=0$ for all $b \in B$.

(3) $n\left[e_{n}\right] \leq\left[1_{B}\right]$ in $K_{0}(B)$

(4) If $L_{n}: B \rightarrow B$ is defined by $L_{n}(b)=e_{n}$ be $e_{n}$, then $L_{n, \sharp}(x) \equiv x$ for all $x$ in

$\operatorname{Tors} K_{0}(A) \oplus K_{1}(A) \oplus \bigoplus_{m=2}^{\infty} K_{*}(A ; \mathbb{Z} / m)$.

Proof. This is contained in [10, Lemma 6.5] (or [32] in the nuclear case), except for part (4). As a consequence of (3) we have

$$
x \equiv L_{n \sharp}(x)+n ! \mu_{n \sharp}(x), \quad \forall x \in \underline{\mathrm{K}}(B .)
$$

Let us argue that (4) follows from this. Indeed, for $x \in K_{1}(B)$ this is the case since $K_{1}\left(F_{n}\right)=$ 0 , hence $\mu_{n \sharp}(x) \equiv 0$. If $x \in \operatorname{Tors}_{0}(A) \oplus \bigoplus_{m=2}^{\infty} K_{*}(A ; \mathbb{Z} / m)$, then $n ! \mu_{n \sharp}(x) \equiv 0$ hence $x \equiv L_{n \sharp}(x)$.

Lemma 8.5. Let $B$ be an infinite dimensional separable simple unital tracially $A F C^{*}$ algebra and let $Z$ be a space on the list (46). If $\alpha \in \mathrm{KK}\left(C_{0}(Z), B\right)$ maps $K_{0}\left(C_{0}(Z)\right)$ into $\operatorname{Inf} K_{0}(B)$, then for any nonzero projection $p \in B$ there is a ccp asymptotic morphism $\left(\varphi_{n}\right)$ : $C_{0}(Z) \rightarrow p B p$ such that $\varphi_{n \sharp}(x) \equiv \alpha_{*}(x)$ for all $x \in \underline{\mathrm{K}}\left(C_{0}(Z)\right)$.

Proof. By Corollary 5.7 there is a ccp asymptotic morphism $\left(\varphi_{n}^{\prime}\right)$ with $\varphi_{n}^{\prime}: C_{0}(Z) \rightarrow$ $M_{k(n)}(B)$ such that $\varphi_{n \sharp}^{\prime}(x) \equiv \alpha_{*}(x)$ for all $x \in \underline{K}\left(C_{0}(Z)\right)$. If $\left(e_{n}\right)$ and $\left(L_{n}\right)$ are as in Proposition 8.4, then by passing to a suitable subsequence $\left(e_{s(n)}\right)$, with $k(n)\left[e_{s(n)}\right] \leq[p]$, we can arrange that $\left(\varphi_{n}\right)=\left(\left(i d_{k(n)} \otimes L_{s(n)}\right) \circ \varphi_{n}^{\prime}\right)$ is a ccp asymptotic morphism which maps $C_{0}(Z)$ into $p B p$. Since $\left(L_{n}\right)$ satisfies the condition (4) of Proposition 8.4, we can also arrange that $\varphi_{n \sharp}(x) \equiv \alpha_{*}(x)$ for all $x \in \operatorname{Tors} K_{0}\left(C_{0}(Z)\right) \oplus K_{1}\left(C_{0}(Z)\right) \oplus \bigoplus_{m=2}^{\infty} K_{*}\left(C_{0}(Z) ; \mathbb{Z} / m\right)$. This proves the statement if $Z$ is the 3 -dimensional simplex, and if $Z=S^{1}, Z=W_{m}$ or $Z=S W_{m}$ since in all these cases $K_{0}\left(C_{0}(Z)\right)$ is a torsion group. It remains to deal with the case $Z=S^{2}$. Let $z$ be a generator of $K_{0}\left(C_{0}\left(S^{2}\right)\right)=\mathbb{Z}$. By Corollary 5.7, there are ccp asymptotic morphisms $\varphi_{n}^{\prime}: C_{0}\left(S^{2}\right) \rightarrow M_{r(n)}(\mathbb{C})$ and $\varphi_{n}^{\prime \prime}: C_{0}\left(S^{2}\right) \rightarrow M_{t(n)}(\mathbb{C})$ such that $\varphi_{n \sharp}^{\prime}(z) \equiv\left[e^{\prime}\right]=1$ and $\varphi_{n \sharp}^{\prime \prime}(z) \equiv-\left[e^{\prime \prime}\right]=-1$, where $e^{\prime}$ and $e^{\prime \prime}$ are minimal projections in $M_{r(n)}(\mathbb{C})$ and $M_{t(n)}(\mathbb{C})$, respectively. Let $x=\alpha_{*}(z) \in \operatorname{Inf} K_{0}(B)$. By Lemma 8.3, there are projections $p_{n}, q_{n}$ in $B$ such that $x=\left[p_{n}\right]-\left[q_{n}\right]$ and $\widehat{p}_{n} \rightarrow 0, \widehat{q}_{n} \rightarrow 0$. After passing to suitable subsequences we may arrange that $r(n)\left[p_{n}\right]+t(n)\left[q_{n}\right] \leq[p]$. Therefore we find *-homomorphisms $\psi_{n}^{\prime}: M_{r(n)}(\mathbb{C}) \rightarrow p B p$ and $\psi_{n}^{\prime \prime}: M_{t(n)}(\mathbb{C}) \rightarrow p B p$ with orthogonal ranges and such that $\psi_{n \sharp}^{\prime}\left[e^{\prime}\right]=\left[p_{n}\right]$ and $\psi_{n \sharp}^{\prime \prime}\left[e^{\prime \prime}\right]=\left[q_{n}\right]$. Then $\varphi_{n}=\psi_{n}^{\prime} \varphi_{n}^{\prime}+\psi_{n}^{\prime \prime} \varphi_{n}^{\prime \prime}$ defines a ccp asymptotic morphism from $C_{0}\left(S^{2}\right)$ to $p B p$ with $\varphi_{n \sharp}(z)=x$. 
Corollary 8.6. Let $B$ be an infinite dimensional separable simple unital tracially $A F C^{*}$ algebra and let $X$ be a model space. Then $C(X) \stackrel{K}{\rightsquigarrow} B$.

Proof. We identify $\mathrm{KK}(C(X), B)$ with $\operatorname{Hom}_{\Lambda}(\underline{K}(C(X)), \underline{K}(B))$ by Theorem 2.1. Let $\alpha \in$ $\mathrm{KK}(C(X), B)$ with $\alpha_{*}\left(K_{0}(C(X))^{+}\right) \subset K_{0}(B)^{+}$. Let $q \in \operatorname{Proj}(B)$ with $\alpha_{*}[1]=[q]$. After replacing $q M_{N}(B) q$ by $B$, one may assume that $\alpha_{*}[1]=\left[1_{B}\right]$. We need to show that there is a ucp asymptotic morphism from $C(X)$ to $B$ which induces $\alpha$. The space $X$ is of the form $X=Z_{0} \vee \cdots \vee Z_{r}, \quad r \geq 0$, with $Z_{i}$ spaces from the list (46) for $i \geq 1$. Let $\alpha^{\prime} \in$ $K K\left(C_{0}(X), B\right)$ denote the restriction of $\alpha$. Viewing $C_{0}\left(Z_{i}\right)$ as subalgebras of $C_{0}(X)$, we write $\alpha^{\prime}=\sum_{i} \alpha_{i}$ with $\alpha_{i} \in \mathrm{KK}\left(C_{0}\left(Z_{i}\right), B\right)$. Since $\alpha$ is positive, it preserves the infinitesimals and so $\left(\alpha_{i}\right)_{*}\left(K_{0}\left(C_{0}\left(Z_{i}\right)\right) \subset \alpha_{*}\left(\operatorname{Inf} K_{0}(C(X))\right) \subset \operatorname{Inf} K_{0}(B)\right.$. By Lemma 8.5, if $p_{i} \in B$ are mutually orthogonal projections with $\sum_{i} p_{i} \leq 1_{B}$, then there are ccp asymptotic morphisms from $C_{0}\left(Z_{i}\right)$ to $p_{i} B p_{i}$ inducing $\alpha_{i}$. The unitization of the sum of these asymptotic morphisms gives a ucp asymptotic morphism from $C(X)$ to $B$ with the desired property.

The following result is essentially due to Lin.

Proposition 8.7. Let $A$ be a model $A H$ algebra and let $B$ be a simple unital tracially $A F$ $C^{*}$-algebra. Then $A \stackrel{K}{\rightsquigarrow} B$.

Proof. The result follows from Proposition 8.6 and Lemma 8.2 if $B$ is infinite dimensional. If $B$ is finite dimensional the statement is trivial.

\section{REFERENCES}

1. B. Blackadar, K-Theory for Operator Algebras, MSRI Monographs, vol. 5, Springer Verlag, Berlin and New York, 1986.

2. B. Blackadar and D. Handelman, Dimension functions and traces on $C^{*}$-algebras, J. Funct. Anal. 45 (1982), 297-340.

3. B. Blackadar and E. Kirchberg, Generalized inductive limits of finite-dimensional $C^{*}$-algebras, Math. Ann. 307 (1997), no. 3, 343-380.

4. N. P. Brown, Herrero's approximation problem for quasidiagonal operators, J. Funct. Anal. 186 (2001), no. $2,360-365$.

5. M. Dadarlat, On the topology of the Kasparov groups and its applications, preprint 2004.

6. Reduction to dimension three of local spectra of real rank zero $C^{*}$-algebras, J. Reine Angew. Math. 460 (1995), 189-212.

7. _ Nonnuclear subalgebras of AF algebras, Amer. J. Math. 122 (2000), no. 3, 581-597.

8. _ Residually finite dimensional $C^{*}$-algebras and subquotients of the CAR algebra, Math. Research Letters 8 (2001), 545-555.

9. S_ Some remarks on the universal coefficient theorem in $K K$-theory, Operator algebras and mathematical physics (Constanţa, 2001), Theta, Bucharest, 2003, pp. 65-74.

10. M. Dadarlat and S. Eilers, On the classification of nuclear $C^{*}$-algebras, Proc. London Math. Soc. (3) 85 (2002), no. 1, 168-210.

11. M. Dadarlat and T. A. Loring, K-homology, asymptotic representations, and unsuspended E-theory, J. Funct. Anal. 126 (1994), no. 2, 367-383.

12. __ A universal multicoefficient theorem for the Kasparov groups, Duke Math. J. 84 (1996), no. 2, $355-377$.

13. M. Dadarlat, G. Nagy, A. Némethi, and C. Pasnicu, Reduction of topological stable rank in inductive limits of $C^{*}$-algebras, Pacific J. Math. 153 (1992), 267-276.

14. M. Dadarlat and A. Némethi, Shape theory and (connective) K-theory, J. Operator Theory 23 (1990), no. 2, 207-291.

15. Marius Dadarlat, Residually finite-dimensional $C^{*}$-algebras, Operator algebras and operator theory (Shanghai, 1997), Contemp. Math., vol. 228, Amer. Math. Soc., Providence, RI, 1998, pp. 45-50. 
16. E. G. Effros, D. E. Handelman, and C.-L. Shen, Dimension groups and their affine representations, American J. Math. 102 (1980), 385-407.

17. G. A. Elliott, On the classification of $C^{*}$-algebras of real rank zero, J. Reine Angew. Math. 443 (1993), 179-219.

18. _ The classification problem for amenable $C^{*}$-algebras, Proceedings of the International Congress of Mathematicians (Basel), vol. 1,2, Birkhäuser, 1995, pp. 922-932.

19. G. A. Elliott and G. Gong, On the classification of $C^{*}$-algebras of real rank zero, II, Ann. of Math. 144 (1996), 497-610.

20. G. Gong, On inductive limits of matrix algebras over higher dimensional spaces, part I, Math. Scand. 80 (1997), 41-55.

21. _ On inductive limits of matrix algebras over higher dimensional spaces, part II, Math. Scand. 80 (1997), 56-100.

22. K. R. Goodearl and D. Handelman, Rank functions and $K_{0}$ of regular rings, J. Pure Appl. Algebra 7 (1976), 195-216.

23. K. R. Goodearl and D. E. Handelman, Metric completions of partially ordered abelian groups, Indiana Univ. Math. J. 29 (1980), no. 6, 861-895.

24. G. G. Kasparov, Hilbert $C^{*}$-modules: Theorems of Stinespring and Voiculescu, J. Operator Theory 4 (1980), 133-150.

25. E. Kirchberg, The classification of purely infinite $C^{*}$-algebras using Kasparov's theory, preprint, 1994.

26. __ Exact $\mathrm{C}^{*}$-algebras, tensor products, and the classification of purely infinite algebras, Proceedings of the International Congress of Mathematicians, Vol. 1, 2 (Zürich, 1994) (Basel), Birkhäuser, 1995, pp. 943-954.

27. A. Kishimoto, Non-commutative shifts and crossed products, J. Funct. Anal. 200 (2003), no. 2, 281-300.

28. H. Lin, Classification of simple $C^{*}$-algebras of tracial topological rank zero, MSRI preprint, 2000.

29. _ Classification of simple tracially AF $C^{*}$-algebras, Canad. J. Math. 53 (2001), no. 1, 161-194.

30. $ـ$ Residually finite dimensional and AF-embeddable $C^{*}$-algebras, Proc. Amer. Math. Soc. 129 (2001), no. 6, 1689-1696.

31. The tracial topological rank of $C^{*}$-algebras, Proc. London Math. Soc. (3) 83 (2001), no. 1, 199-234.

32. _ Tracially AF $C^{*}$-algebras, Trans. Amer. Math. Soc. 353 (2001), no. 2, 693-722.

33. Classification of simple $C^{*}$-algebras and higher dimensional noncommutative tori, Ann. of Math. (2) 157 (2003), no. 2, 521-544.

34. N. Ozawa, Homotopy invariance of AF-embeddability, Geom. Funct. Anal. 13 (2003), no. 1, 216-222.

35. N. C. Phillips, A Classification Theorem for Nuclear Purely Infinite Simple $C^{*}$-Algebras, Documenta Math. (2000), no. 5, 49-114.

36. N. Christopher Phillips, When are crossed products by minimal diffeomorphisms isomorphic?, Operator algebras and mathematical physics (Constanţa, 2001), Theta, Bucharest, 2003, pp. 341-364.

37. S. Popa, On locally finite dimensional approximation of $C^{*}$-algebras, Pacific J. Math. (1997), 141-158.

38. M. Rørdam, Classification of nuclear, simple $C^{*}$-algebras, Encyclopaedia Math. Sci., vol. 126, Springer, Berlin, 2002.

39. M. Rørdam, A simple $C^{*}$-algebra with a finite and an infinite projection, Acta Math. 191 (2003), no. 1, 109-142.

40. J. Rosenberg and C. Schochet, The Künneth Theorem and the Universal Coefficient Theorem for Kasparov's generalized K-functor, Duke Math. J. 55 (1987), no. 2, 431-474.

41. C. Schochet, Topological methods for $C^{*}$-algebras. IV. Mod p homology, Pacific J. Math. 114 (1984), no. $2,447-468$.

42. G. Skandalis, Une Notion de Nucléarité en K-théorie, K-theory 1 (1988), no. 5, 549-573.

43. D. Voiculescu, A non-commutative Weyl-von Neumann theorem, Rev. Roum. Math. Pures et Appl. 21 (1976), 97-113.

44. _ A note on quasidiagonal $C^{*}$-algebras and homotopy, Duke Math. J. 62 (1991), no. 2, 267-271.

45. Shuang Zhang, A Riesz decomposition property and ideal structure of multiplier algebras, J. Operator Theory 24 (1990), no. 2, 209-225. 
Department of Mathematics, Purdue University, West Lafayette IN 47907

E-mail address: mdd@math.purdue.edu 